\title{
Singular Nature of the Elastocapillary Ridge
}

\author{
A. Pandey $\odot,{ }^{1,2}$ B. Andreotti, ${ }^{3}$ S. Karpitschka, ${ }^{4}$ G. J. van Zwieten, ${ }^{5}$ E. H. van Brummelen $\odot,{ }^{6}$ and J. H. Snoeijer ${ }^{1}$ \\ ${ }^{1}$ Physics of Fluids Group, Faculty of Science and Technology, Mesa+Institute, \\ University of Twente, 7500 AE Enschede, Netherlands \\ ${ }^{2}$ Department of Biological and Environmental Engineering, Cornell University, \\ Ithaca, New York 14853, USA \\ ${ }^{3}$ Laboratoire de Physique de l'ENS, UMR 8550 Ecole Normale Supérieure, CNRS, \\ Université de Paris, Sorbonne Université, 24 rue Lhomond, 75005 Paris \\ ${ }^{4}$ Max Planck Institute for Dynamics and Self-Organization (MPIDS), 37077 Göttingen, Germany \\ ${ }^{5}$ Evalf Computing, Burgwal 45, 2611 GG Delft, Netherlands \\ ${ }^{6}$ Multiscale Engineering Fluid Dynamics Group, Department of Mechanical Engineering, \\ Eindhoven University of Technology, P.O. Box 513, 5600 MB Eindhoven, Netherlands
}

(Received 19 March 2020; revised 20 June 2020; accepted 5 August 2020; published 25 September 2020)

\begin{abstract}
The mechanical and chemical properties of soft solids are crucial to many applications in biology and surface science. Recent studies use wetting by liquid drops to probe the surface mechanics of reticulated polymer networks, leading to controversial interpretations. This controversy relates to the long-standing paradox of Young's law for the liquid contact angle, which invokes only a horizontal force balance. Recent work shows that, for very soft materials, the solid's surface tension plays a key role for the vertical force balance, involving a singular ridgelike deformation exactly at the point where the droplet pulls on the network. A hotly debated question is whether unexpected measurements on this singular deformation can be attributed to nonlinear bulk elasticity or whether these provide evidence for an intricate surface elasticity, known as the Shuttleworth effect. Here, we theoretically reveal the nature of the elastocapillary singularity on a hyperelastic substrate with various constitutive relations for the interfacial energy. First, we finely resolve the vicinity of the singularity using goal-adaptive finite-element simulations. This simulation confirms that bulk elasticity cannot affect the force balance at the contact line. Subsequently, we derive exact solutions of nonlinear elasticity that describe the singularity analytically. These solutions are in perfect agreement with numerics and show that both the angles and stretch at the contact line, as previously measured experimentally, consistently point to a strong Shuttleworth effect. Finally, using Noether's theorem, we reveal the quantitative link between Young's law, hysteresis, and the nature of the elastocapillary singularity. Our contribution closes the issue of the missing normal force at the contact line and opens up the development of modern techniques in polymer surface science.
\end{abstract}

DOI: 10.1103/PhysRevX.10.031067

Subject Areas: Soft Matter

\section{INTRODUCTION}

The wetting and adhesion of soft materials have recently become a quickly expanding domain, finding applications in the design of innovative materials (adhesives [1], slippery surfaces [2], and highly stretchable electronics [3]), to analyze the mechanics of cells and biological tissues $[4,5]$, and in between, in the field of bioengineering (reversible adhesives [6], e-skin [7], etc.). Reticulated polymers, composed of long molecular chains forming a network, are model soft materials with versatile properties.

Published by the American Physical Society under the terms of the Creative Commons Attribution 4.0 International license. Further distribution of this work must maintain attribution to the author(s) and the published article's title, journal citation, and DOI.
At small length and timescales, their structure is liquidlike and highly deformable. At large scales, however, the presence of cross-links gives the polymer networks a finite shear modulus $G$ such that they behave like elastic solids [8-11]. The elasticity is of entropic origin, and, as a consequence, the elastic moduli of polymer networks can be exceedingly small compared to those of (poly) crystalline materials, whose elasticity is of enthalpic origin. Rubbers and edible hydrogels such as gelatin desserts are everyday examples of soft solid materials that exhibit entropic elasticity.

The dual liquid-and-solid character plays a central role when the polymeric solid is wetted by liquid drops. The overall shape of the wetting liquid remains spherical, similar to drops on a (stiff) glass window. In the field of wetting, it is common to infer the surface energies of the system by measuring the contact angle: The liquid-vapor 

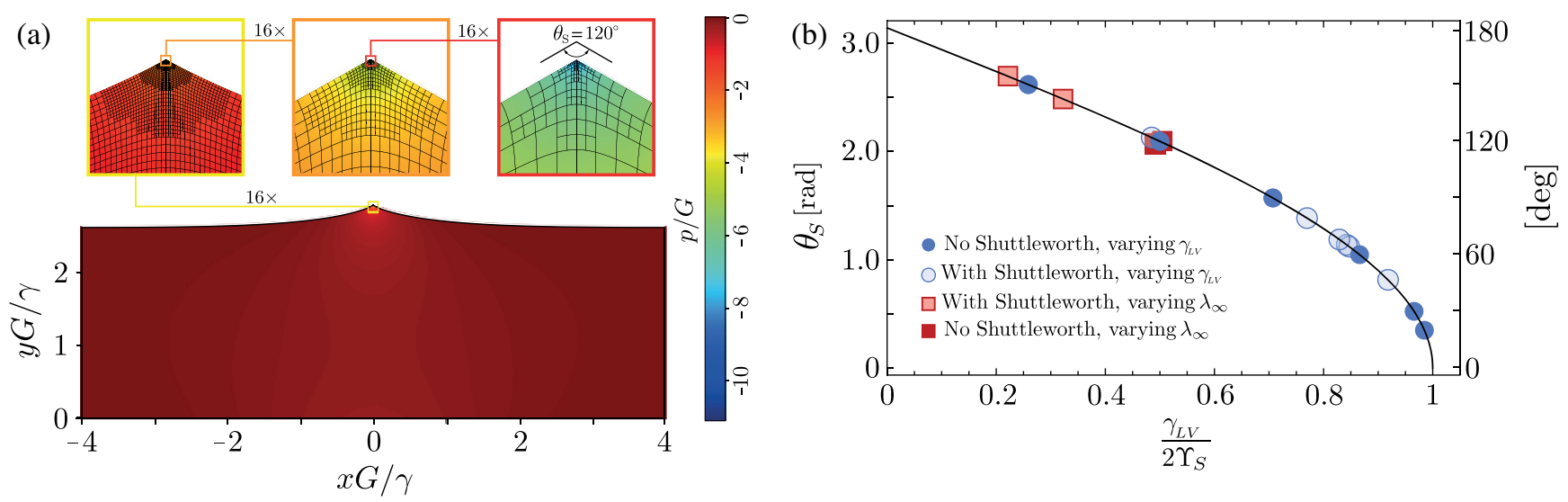

FIG. 1. Symmetric wetting ridges under large deformation induced by a localized contact line force, with and without the Shuttleworth effect. (a) Typical numerical solution using a finite-element method, where successive magnifications show the adaptive resolution of the elastocapillary ridge. The example is a case without the Shuttleworth effect, with equal liquid and solid surface energies $\gamma$ (giving a solid angle $\theta_{S}=120^{\circ}$ ). The scales are expressed in the corresponding elastocapillary length $\gamma / G$. (b) The solid angle $\theta_{S}$ versus the ratio of liquid-vapor surface tension $\gamma_{\mathrm{LV}}$ and solid surface tension $\Upsilon_{S}$. Symbols are numerical results with the Shuttleworth effect (open symbols, $\Upsilon_{S}$ measured at the contact line) and without the Shuttleworth effect (closed symbols). We vary both $\gamma_{\mathrm{LV}}$ (circles) and the amount of prestretch of the substrate from $\lambda_{\infty}=1$ to 2 (squares). The solid line corresponds to Neumann's law (15), with $\Upsilon_{S}$ based on its value at the contact line.

interface reaches the edge of the drop at an angle $\theta_{L}$ with respect to the solid. The droplet's edge represents a threephase contact line, where, respectively, the liquid-vapor (LV), solid-liquid (SL), and solid-vapor (SV) interfaces meet. On stiff solids, the resulting liquid angle is given by Young's law [12]:

$$
\gamma_{\mathrm{LV}} \cos \theta_{L}+\gamma_{\mathrm{SL}}-\gamma_{\mathrm{SV}}=0
$$

where $\gamma_{\alpha \beta}$ denote the surface free energies of the three interfaces. Classically, Young's law can be interpreted as a horizontal force balance in the vicinity of the three-phase contact line. On a soft substrate, however, the vertical force balance becomes nontrivial and involves a singular elastic deformation near the contact line. Indeed, intermolecular forces are able to deform the soft solid over a scale set by the balance between capillarity and elasticity (quantified by the static shear modulus $G$ ), known as the elastocapillary length [13-19]:

$$
\ell=\frac{\gamma_{\mathrm{LV}}}{G} .
$$

Below this length scale, the soft substrate takes the shape of a sharp ridge, culminating at the contact line-Fig. 1(a) shows a typical example of such an elastocapillary ridge, introduced by a localized force. Thus, this sessile-droplet experiment of soft wetting gives rise to many features which have no counterpart in the wetting of rigid solids and, naturally, raises a number of fundamental questions: Is the liquid contact angle with respect to the undeformed substrate still selected by the Young's law? Which forces are acting on this contact line, manifesting themselves in the solid opening angle $\theta_{S}$ [see Fig. 1(a)]? Is the local structure of the interfaces at the contact line selected by a simple force balance, leading to a generalized Neumann's law? Is the contact lined pinned to a material point of the solid, or can it freely move despite the singular deformation underneath it?

Soft wetting, in particular, the questions raised above, has recently led to a strong controversy on the nature of the forces that counteract localized surface perturbations. Experiments demonstrate that the solid angle $\left(\theta_{S}\right)$ and local stretch at the ridge tip can be manipulated by a global stretching of the substrate [20]. Two different explanations are presented to interpret these surprising experimental results. On the one hand, it is proposed that bulk elasticity may present singular contributions at a contact line [21,22]. These are known as Eshelby forces, which are classically used to describe defects in crystalline solids [23]. On the other hand, these experiments are interpreted by introducing a pure surface-elastic contribution: the so-called Shuttleworth effect [24-27], which makes the capillary forces at an elastic interface different from a simple fluid interface. In principle, the surface energy $\gamma$ of a solid can depend on the surface stretch $\lambda$. In this case, surface energy $\gamma$ and surface tension $\Upsilon$ are no longer equal but obey the Shuttleworth relation [24]:

$$
\Upsilon=\gamma+\lambda \frac{d \gamma}{d \lambda} .
$$

This result offers an exciting perspective analogous to the surface rheology of soap films, where surface tension $\Upsilon$ depends on the state $\lambda$ of the system - potentially leading to stiffening or even softening of the interface. However, 
given that interfacial properties are determined at the nanoscale, where soft polymeric networks behave very much like simple fluids, the emergence of a Shuttleworth effect for these materials is debated [20-22,27-35]. To a large extent, the discussion is due to a lack of a consistent analytical theory to interpret macroscopic experiments.

The controversies on the existence, or not, of the Shuttleworth effect or Eshelby forces in soft solids revolve around these questions, but a consistent theory relating forces and geometry is missing to date [36]. Thus, the interpretation of recent experimental $[20,30,31]$ and numerical $[21,22,33]$ work diverges. Before trying to analyze the microscopic origin of potential Eshelby or Shuttleworth effects, there is an urgent need to clarify the mechanical consequences of the existence of such effects. In particular, numerical simulations ultimately rely on a mechanical description which must be totally selfconsistent, including the possibility of singularities.

In this paper, we numerically resolve the problem of soft wetting, using an adaptive numerical technique that allows us to resolve the elastocapillary wetting ridge on all scales [Fig. 1(a)]. The approach is based on minimization of elastocapillary energy for a substrate subjected to a perfectly localized contact line force. The method includes the possibility of singularities, large elastic deformations, and the Shuttleworth effect. It is found that the elastic singularity at the wetting ridge is not sufficiently strong to interfere with the balance of surface tensions at the contact line, so that Neumann's law is valid in all circumstancesirrespective of the presence of large deformations, the Shuttleworth effect, or pinning. Subsequently, we provide the exact asymptotic solution that describes the nonlinear elastic deformation near the ridge tip. Based on the numerical and analytical results, we offer a novel route to interpret experiments, via the surface stretch measured at the contact line, which provides further independent evidence for a strong Shuttleworth effect in elastomers.

The paper is organized as follows. The theoretical framework and the adaptive numerical method are described in Sec. II, and the numerical results are presented in detail in Sec. III. Subsequently, in Sec. IV, we derive exact solutions to nonlinear elasticity that analytically resolve the ridge singularity in the presence of large deformations. These asymptotic solutions, valid near the singularity, are fully confirmed by the numerical results. Finally, we show in Sec. V how Eshelby-like forces can emerge when the substrate has true defects that represent pinning sites on the substrate and reveal their effect on the contact angles. The paper closes with a critical overview of the state of the art in Sec. VI and by a discussion of the broader implications of our findings in Sec. VII.

\section{FREE ENERGY FORMULATION}

In experiments, the drop size is usually large compared to the elastocapillary length $\gamma / G$, where $\gamma$ is a typical surface energy (of the solid or the liquid) while $G$ is the shear modulus of the substrate. In this regime, the curvature of the contact line is negligible compared to the size of the wetting ridge, and the geometry is quasi-two-dimensional. Below, we therefore formulate the problem in a plane strain description and, subsequently, explain the numerical method that is used to adaptatively resolve the singular nature of the elastocapillary ridge. The results of the simulations are presented in Sec. III.

\section{A. Minimizing the elastocapillary energy}

The statics of wetting amounts to finding the state of minimal elastocapillary energy. The substrate deformation is described by a mapping from the reference state prior to deformation to a current state after deformation. Following standard notation, the mapping is written as

$$
\mathbf{x}=\chi(\mathbf{X}),
$$

where $\mathbf{X}$ is the position of a material point on the reference domain, mapped onto its current position $\mathbf{x}$. We consider the geometry to be invariant along the contact line, so that the problem is two-dimensional (plane strain elasticity). Hyperelastic solids are described by an elastic energy density $W(\mathbf{F})$, which depends on the deformation gradient tensor $\mathbf{F}=\partial \mathbf{x} / \partial \mathbf{X}$. We now turn to the interface, which in the (plane strain) two-dimensional description is onedimensional. We define the arc-length material coordinate at the interface as $S$, and the current surface position $\mathbf{x}_{s}(S)=\chi[\mathbf{X}(S)]$. The surface stretch, accounting for the change of length of surface elements, follows as

$$
\lambda^{2}=\frac{\partial \mathbf{x}_{s}}{\partial S} \cdot \frac{\partial \mathbf{x}_{s}}{\partial S},
$$

which is a scalar in this plane strain description. Now we can express capillarity, usually defined by the excess energy $\gamma$ per unit area of the deformed state, as a free energy $\lambda \gamma$ per unit area in the reference state.

Crucially, elastic media can exhibit a nontrivial capillarity where the surface energy $\gamma(\lambda)$ is itself a function of the stretch $\lambda$-the Shuttleworth effect [24-27]. With this result, the elastocapillary energy (per unit length along the contact line) takes the form

$$
\mathcal{E}[\chi]=\int d^{2} X W(\mathbf{F})+\oint d S \lambda \gamma(\lambda),
$$

respectively giving the total (bulk) elastic energy and the (surface) capillary energy. $\mathbf{F}$ and $\lambda$ are the corresponding bulk and surface stretches, respectively, and are both defined by the map $\chi(\mathbf{X})$. We anticipate that we will consider incompressible substrates, in which case the constraint of incompressibility is included in $W(\mathbf{F})$. 
Equilibrium configurations of the elastocapillary substrate are found by minimizing the functional $\mathcal{E}$ with respect to $\chi(\mathbf{X})$. Considering variations $\delta \mathbf{x}=\delta \chi(\mathbf{X})$, we find

$$
\begin{aligned}
\delta \mathcal{E} & =\int d^{2} X\left(\frac{\partial W}{\partial \mathbf{F}}: \delta \mathbf{F}\right)+\oint d S \frac{d(\lambda \gamma)}{d \lambda} \delta \lambda \\
& =\int d^{2} X[\mathbf{s}: \operatorname{Grad}(\delta \mathbf{x})]+\oint d S\left(\Upsilon \mathbf{t} \cdot \frac{\partial \delta \mathbf{x}}{\partial S}\right) .
\end{aligned}
$$

Here, we introduce the nominal (or first Piola-Kirchhoff) stress tensor $\mathbf{s}$ and the surface tension $\Upsilon$ :

$$
\mathbf{s}=\frac{\partial W}{\partial \mathbf{F}}, \quad \Upsilon=\frac{d(\lambda \gamma)}{d \lambda}=\gamma+\lambda \frac{d \gamma}{d \lambda},
$$

where for the latter we indeed recognize the Shuttleworth relation (3). In addition, we use that $\delta \lambda=\mathbf{t} \cdot \partial \delta \mathbf{x} / \partial S$ along the boundary, where $\mathbf{t}$ is the surface-tangent unit vector in the current configuration.

To study the elastocapillary ridge, we still need to include the pull of the contact line, induced by the liquid drop that is wetting the solid. This inclusion can be achieved by making explicit the capillary energy of the drop, via its liquid-vapor surface energy $\gamma_{\mathrm{LV}}$. The subtlety here is that one needs to impose a constraint at the contact line [32,37]: The position $\mathbf{x}$ of the liquid-vapor interface must (by definition) coincide with that of the solid interface. The effect of this constraint, imposed by a Lagrange multiplier, provides a localized traction on the substrate, pulling with a strength $\gamma_{\mathrm{LV}}$ along the direction of the liquidvapor interface $\mathbf{t}_{\mathrm{LV}}$ [38]. The representation by a local force is indeed commonly used in modeling approaches $[21,22,39-41]$. Here, we therefore treat the contact line as a perfectly localized external traction, with the associated work functional $\mathcal{R}=\gamma_{\mathrm{LV}} \mathbf{t}_{\mathrm{LV}} \cdot \mathbf{x}\left(\mathbf{X}_{\mathrm{cl}}\right)$, where $\mathbf{X}_{\mathrm{cl}}$ is the solid's material point at which the contact line is acting. During the variation, this traction corresponds to a work

$$
\delta \mathcal{R}=\gamma_{\mathrm{LV}} \mathbf{t}_{\mathrm{LV}} \cdot \delta \mathbf{x}\left(\mathbf{X}_{\mathrm{cl}}\right) .
$$

The virtual work principle $\delta \mathcal{E}=\delta \mathcal{R}$ then gives the equilibrium condition

$$
\begin{aligned}
& \int d^{2} X[\mathbf{s}: \operatorname{Grad}(\delta \mathbf{x})]+\oint d S\left(\Upsilon \mathbf{t} \cdot \frac{\partial \delta \mathbf{x}}{\partial S}\right) \\
& =\gamma_{\mathrm{LV}} \mathbf{t}_{\mathrm{LV}} \cdot \delta \mathbf{x}\left(\mathbf{X}_{\mathrm{cl}}\right),
\end{aligned}
$$

which should be satisfied for arbitrary $\delta \mathbf{x}$.

Equation (10) defines the elastocapillary equilibrium in the weak formulation. This equilibrium is indeed highly singular. Namely, the forcing on the right-hand side appears as a point force, pulling at $\mathbf{X}_{\mathrm{cl}}$, while the elastocapillary energies on the left contain only surface and bulk contributions. The debate in the literature precisely revolves around the following question: Do singularities appear in the surface (capillarity) or in the bulk (elasticity), in order to balance the point force at the contact line?

\section{B. Numerical method}

Our interest pertains to finding equilibrium configurations of the elastocapillary problem, i.e., to minimizers of the energy functional in Eq. (6) extended with the work functional $\mathcal{R}$ representing the contact line, subject to appropriate boundary conditions. Specifically, we consider substrates that are flat in the reference configuration, with complete fixation at the bottom boundary and guided fixation (slip) at the lateral boundaries. We allow for the possibility to impose a prestretch $\lambda_{\infty}$, referring to the uniaxial stretch far away from the contact line. Besides the work associated to the point forcing at the contact line, the top surface is free of traction, as is made explicit in the weak formulation (10) of the minimization problem. The constitutive relations for the strain-energy density and the surface energy are specified in Sec. III below. In all simulations, the shear modulus $G$ and the relevant surface energies are chosen such that the wetting ridge is much smaller than the width of the domain, with a typical example given in Fig. 1(a). In that example, the domain width and height, respectively, are $8 \gamma_{\mathrm{LV}} / G$ and $\frac{8}{3} \gamma_{\mathrm{LV}} / G$, which are representative for all presented results.

Here, we numerically approximate the minimizer of $\mathcal{E}-\mathcal{R}$ by means of a goal-adaptive finite-element method $[42,43]$. In goal-adaptive methods, the finite-element approximation is locally refined on the basis of an a posteriori error estimate, in such a manner that an optimal approximation to a predefined quantity of interest (the goal/) is obtained. Goal-adaptive finite-element methods generally proceed according to the solve $\rightarrow$ estimate $\rightarrow$ mark $\rightarrow$ refine (SEMR) process $[44,45]$. The SEMR process starts by solving a finite-element approximation on a coarse mesh. Next, the contribution of each element to the error in the goal quantity is estimated, based on a so-called dual problem $[42,43,45]$. The elements that yield the largest contribution to the error are marked according to a refinement strategy. These marked elements are subsequently refined by subdivision. This process is repeated until a certain threshold for the error estimate is satisfied or a preset number of refinement iterations is executed. In accordance with our interest in minimizers of $\mathcal{E}-\mathcal{R}$, we take the energy itself as the goal functional. The optimality conditions are resolved by means of the Newton-Raphson method. The goal-adaptive finite-element method for the present problem is implemented in the open-source software framework Nutils [46]. The optimality conditions (10) are, in fact, directly derived from an implementation of the energy functional $\mathcal{E}-\mathcal{R}$ via the automatic-differentiation functionality in Nutils.

An illustration of a goal-adaptive finite-element approximation is provided in Fig. 1(a). The approximation is based on 16 refinement iterations. Accordingly, the smallest 
elements in the adaptive approximation are $2^{16}$ times smaller than the initial element size. The initial mesh comprises $24 \times 8$ uniform quadrilateral elements, and, correspondingly, the smallest elements are 5-6 orders of magnitude smaller than the elastocapillary length. Importantly, the adaptive procedure automatically introduces the local refinements in the vicinity of the contact line. This refinement pattern is in agreement with the singularity of the pressure toward the contact line, and we extensively verify the numerical convergence of the result. For the result shown in Fig. 1(a), the relative numerical error in the computed value of the solid opening angle $\theta_{S}$ is less than $10^{-6}$.

\section{ELASTOCAPILLARY RIDGES, WITH AND WITHOUT A SHUTTLEWORTH EFFECT}

We now present the adaptively resolved numerical results for the elastocapillary ridge. We consider cases with constant surface energy and with variable surface energy, i.e., without and with a Shuttleworth effect. For the bulk elasticity, we consider materials with a neo-Hookean strain-energy density (using plane strain):

$$
W(\mathbf{F})=\frac{1}{2} G(\mathbf{F}: \mathbf{F}-2)-p(\operatorname{det} \mathbf{F}-1),
$$

where we introduce the pressure $p$ to impose the constraint of incompressibility. In contrast to bulk elasticity, there are no standard constitutive relations for the surface energy of soft solids. Here, we propose a surface energy of the form

$$
\gamma_{S}(\lambda)=\gamma_{0}\left[1-c_{0} \log \lambda+c_{1}(\lambda-1)\right] .
$$

We from now on add the subscript " $S$ " to indicate that we refer to the solid interface (to distinguish from the liquidvapor surface energy $\gamma_{\mathrm{LV}}$ ). Expanding Eq. (12) around $\lambda=1$ up to quadratic order, one recovers the ansatz for surface elasticity as proposed in Ref. [35], while if, in addition, $c_{0}=c_{1}$, one finds a linear surface elasticity as proposed in Ref. [30]. An advantage of the constitutive relation (12) is that the logarithm conveniently keeps the system away from $\lambda \rightarrow 0$. The parameters $c_{0,1}$ must satisfy an admissibility condition such that the surface energy remains convex and that both the energy $\gamma_{S}$ and the surface tension $\Upsilon_{S}$ remain positive definite. According to the Shuttleworth relation of Eq. (8), the above surface energy gives a surface tension

$$
\Upsilon_{S}(\lambda)=\gamma_{0}\left[1+c_{1}-c_{0}-c_{0} \log \lambda+2 c_{1}(\lambda-1)\right],
$$

and one verifies that ensuring $\Upsilon_{S}>0$ is sufficient for the constants $c_{0,1}$ to be admissible. Below, we present results for the case where $c_{0,1}=0$ (no Shuttleworth effect) and for $c_{0,1}=1$ (strong Shuttleworth effect), which are indeed in the admissible regime. For later reference, we also define the associated "chemical potential"

$$
\mu_{S}(\lambda) \equiv \lambda^{2} \frac{d \gamma_{S}}{d \lambda}=\gamma_{0}\left(c_{1} \lambda^{2}-c_{0} \lambda\right)
$$

which is relevant in Sec. V.

In general, the solid-liquid and solid-vapor interfaces, of course, exhibit a different surface constitutive relation, respectively, which we write $\gamma_{\mathrm{SL}}(\lambda)$ and $\gamma_{\mathrm{SV}}(\lambda)$. For most of the paper, we focus on cases where the solid-liquid and solid-vapor energies are identical and simply denote $\gamma_{S}(\lambda)$. This focus renders the problem symmetric around the contact line, so that the equilibrium contact angle of the liquid is $90^{\circ}$ and the associated forcing is vertical. Also, this symmetry replaces the "second boundary condition" discussed in Refs. [27,32]. Asymmetric surface energies are considered in Sec. V, where we address the relation between pinning, the contact angle, and the second boundary condition.

\section{A. Universality of Neumann's law}

We first consider the solid angle $\theta_{S}$, as measured at the tip of the wetting ridge in FEM. Figure 1(b) shows $\theta_{S}$ plotted against $\gamma_{\mathrm{LV}} / \Upsilon_{S}$, with the value of $\Upsilon_{S}$ taken at the tip. Clearly, $\theta_{S}$ follows a universal curve for all cases considered. The parameters that are varied in our simulations are the contact line force $\gamma_{\mathrm{LV}}$ [compared to the value of $\gamma_{0}$ in Eq. (12)], while solid surface tensions are with or without a Shuttleworth effect $\left(c_{0,1}=0\right.$ and $c_{0,1}=1$, respectively). We also consider different amounts of prestretch of the substrate, ranging from $\lambda_{\infty}=1$ (no prestretch) to $\lambda_{\infty}=2$ (extending the length by $100 \%$ ). The universal curve for $\theta_{S}$ indeed follows Neumann's law, which for the specific case of identical solid-liquid and solid-vapor energies reads

$$
2 \Upsilon_{S} \sin \left[\frac{1}{2}\left(\pi-\theta_{S}\right)\right]=\gamma_{\mathrm{LV}}
$$

Here, we emphasize that, owing to the Shuttleworth effect, the surface tension $\Upsilon_{S}(\lambda)$ depends on the strain. Since the Neumann balance is to be interpreted as a boundary condition at the contact line, we consider Eq. (15) with values of the stretch $\lambda_{\mathrm{cl}}$ taken at the contact line. The result of Eq. (15) is superimposed as the solid line in Fig. 1(b), providing a perfect description of the FEM results.

We thus reach a first major conclusion: Neumann's law (based on the local values of the surface tension) robustly applies to elastocapillary wetting ridges, irrespective of the large elastic deformations at the contact line. This conclusion rejects the recent hypothesis that strong elastic nonlinearity, as encountered for narrow $\theta_{S}$ and large prestretch, leads to a failure of Neumann's law [22]. This general validity of Neumann's law has an immediate 
consequence for measurements of the surface-constitutive relation based on $\theta_{S}$, since we safely conclude that $\theta_{S}$ gives direct access to the values of $\Upsilon_{S}$. Phrased differently, the experimental observation for Polydimethylsiloxane (PDMS) substrates that $\theta_{S}$ increases with $\lambda_{\infty}$ [20] can, in a macroscopic theory based on hyperelasticity, be explained only via a strong Shuttleworth effect.

To further illustrate this consequence, we closely follow the experimental protocol of Ref. [20] in our simulations and consider how the geometry of the ridge evolves when stretching the substrate by an increasing amount $\lambda_{\infty}$. Figure 2(a) shows $\theta_{S}$ versus the stretch at the contact line $\lambda_{\mathrm{cl}}$. The open circles are FEM results with a Shuttleworth effect $\left(c_{0,1}=1\right.$ and $\left.\gamma_{0}=\gamma_{\mathrm{LV}}\right)$, showing an increase of the solid opening angle $\theta_{S}$. Indeed, the dependence of $\theta_{S}$ is perfectly predicted by Neumann's law (15), as is indicated by the solid line. In experiments, one, of course, does not control the stretch at the contact line $\lambda_{\mathrm{cl}}$ but rather the global stretch of the substrate $\lambda_{\infty}$. In Fig. 2(b), we therefore plot these two stretches against one another. While $\lambda_{\mathrm{cl}}$ is not exactly identical to the imposed stretched $\lambda_{\infty}$, the differences turn out to be minor-consistently with experiments [20]. As a guide to the eye, the dashed line in Fig. 2(b) indicates $\lambda_{\mathrm{cl}}=\lambda_{\infty}$. We expect this near homogeneity of $\lambda$ 's to arise only for nearly symmetric $\gamma_{\mathrm{SL}}$ and $\gamma_{\mathrm{SV}}$, as asymmetry, in general, leads to stronger gradients of stretch (cf. Sec V).

The scenario changes dramatically when the substrate does not exhibit a Shuttleworth effect (i.e., $c_{0,1}=0$ ). In that case, both $\theta_{S}$ and $\lambda_{\mathrm{cl}}$ take on a constant value that is totally independent of the imposed $\lambda_{\infty}$. This result is indicated in Fig. 2(a) by the closed circle-which, in fact, corresponds to various simulations with $\lambda_{\infty}$ ranging from 1 to 2 . This invariance of $\theta_{S}$ with respect to $\lambda_{\infty}$ is easily understood from the Neumann balance. Namely, surface tensions are constant when $c_{0,1}=0$, and, since we consider $\gamma_{0}=\gamma_{\mathrm{LV}}$,

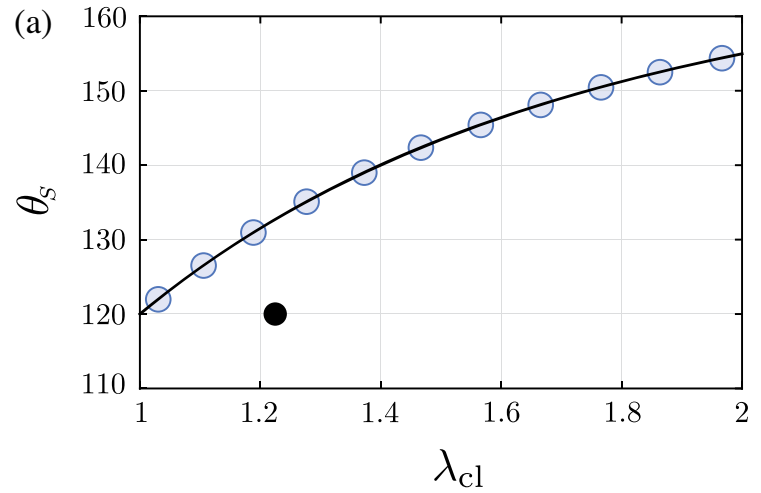

we find that $\theta_{S}=120^{\circ}$. By contrast, the invariance of the stretch at the tip comes as a surprise, and its explanation calls for a better understanding of the nature of the elastic singularity. Below, we derive analytically that, without the Shuttleworth effect, $\lambda_{\mathrm{cl}}=\sqrt{\pi / \theta_{S}}$, irrespective of the externally imposed prestretch $\lambda_{\infty}$ of the substrate.

Measurements of the stretch at the contact line, thus, provide important additional information on the Shuttleworth effect, that to date has not yet been explored. Namely, experiments by Ref. [20] reveal an increase of stretch at the contact line upon a global stretching of the substrate. From the above, it is clear that such a dependence can, in a macroscopic theory based on hyperelasticity, not occur when there is no Shuttleworth effect.

\section{B. Stress singularity and the elastic Marangoni effect}

To further analyze the vicinity of the tip, we now turn to the elastic stress measured along the free surface. In Figs. 3(a) and 3(b), we plot the pressure $p$ as a function of the distance to the contact line $x$, on a semilogarithmic scale. In all cases, the FEM simulations exhibit a weak singularity of the pressure, diverging logarithmically with the distance to the tip.

Figure 3(a) corresponds to a case without a Shuttleworth effect $\left(c_{0,1}=0\right)$, for different ratios $\gamma_{\mathrm{LV}} / \gamma_{0}$. With this case, we cover a broad range of $\theta_{S}$ down to very narrow angles with $20^{\circ}$. The prefactor of the logarithmic pressure singularity is larger for narrow $\theta_{S}$. The pressure plotted in Fig. 3(a) is scaled by $G \times\left[\left(\pi / \theta_{S}\right)-\left(\theta_{S} / \pi\right)\right]$, which indeed captures the $\theta_{S}$ dependence of the prefactor of the singularity. We remark that for very narrow angles the logarithmic asymptotic emerges only at distances much below the elastocapillary length $\gamma_{0} / G$; this result illustrates the challenge of accurate numerical resolutions for small $\theta_{S}$. Figure 3(b) corresponds to the case with a strong

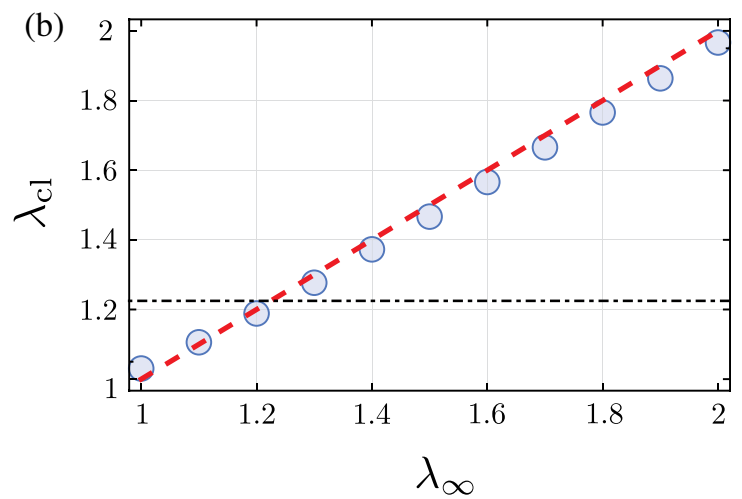

FIG. 2. Geometry of the elastocapillary ridge upon stretching the substrate. (a) Solid angle $\theta_{S}$ as a function of the stretch at the contact line $\lambda_{\mathrm{cl}}$. Open circles correspond to FEM in the presence of a strong Shuttleworth effect $\left(c_{0}=c_{1}=1\right.$, with $\left.\gamma_{0}=\gamma_{\mathrm{LV}}\right)$. The solid line is the analytical prediction by Neumann's law (15). The closed circles (several measurements superimposed) correspond to FEM without a Shuttleworth effect $\left(c_{0}=c_{1}=0\right.$, with $\left.\gamma_{0}=\gamma_{\mathrm{LV}}\right)$. (b) Relation between the stretch at the contact line $\lambda_{\mathrm{cl}}$ and the globally imposed stretch $\lambda_{\infty}$. In the presence of a strong Shuttleworth effect, the two stretches takes on very similar values (the red dashed line, $\lambda_{\mathrm{cl}}=\lambda_{\infty}$, is a guide to the eye). Without a Shuttleworth effect, $\lambda_{\mathrm{cl}}=\sqrt{\pi / \theta_{S}}$ takes on a constant value (dash-dotted line). 

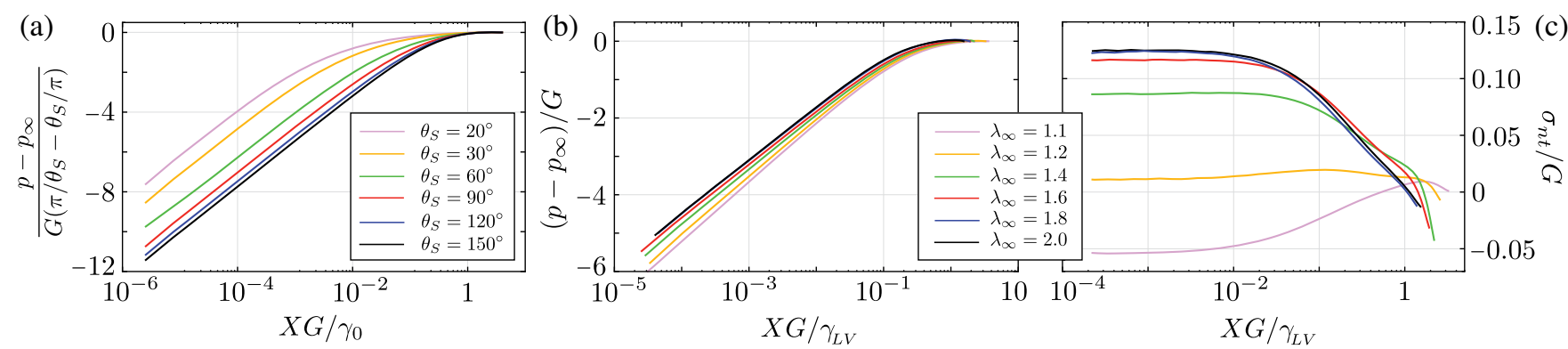

FIG. 3. Elastic stress along the free surface near the ridge singularity (symmetric ridges). (a) Pressure $p$ versus distance to the contact line $X$, scaled as indicated on the axes. Data correspond to the situation without a Shuttleworth $\left(c_{0,1}=0\right)$ with different $\theta_{S}$ obtained by varying the ratio $\gamma_{\mathrm{LV}} / \gamma_{0}$. (b) Pressure $p$ versus distance to the contact line $x$, scaled as indicated on the axes. Data correspond to the situation with a Shuttleworth $\left(c_{0,1}=1\right)$ with different amounts of prestretch $\lambda_{\infty}$. (c) Shear stress $\sigma_{n t}$ versus distance to the contact line $x$, scaled as indicated on the axes. Data correspond to the same cases as in (b).

Shuttleworth effect $\left(c_{0,1}=1\right)$, for different amounts of substrate prestretch $\lambda_{\infty}$ (the corresponding $\theta_{S}$ are in Fig. 2). Figure 3(b) again reveals a logarithmic singularity of pressure, with a weak variation of the prefactor with $\lambda_{\infty}$.

Interestingly, the Shuttleworth effect allows for a new phenomenon induced by gradients of surface tension. For liquid interfaces, gradients in surface tension arise due to gradients in composition or in temperature- this phenomenon is known as the Marangoni effect and leads to tangential interfacial stress. For the elastic interfaces considered here, the gradients in surface tension are due to gradients of $\lambda$ along the interface. Given this analogy, we refer to this phenomenon as the elastic Marangoni effect.

Figure 3(c) indeed reveals the emergence of elastic (Cauchy) shear stress $\sigma_{n t}$ along the interface, which we refer to as elastic Marangoni stress. Somewhat surprisingly, the Marangoni stress is not singular but converges to a constant value upon approaching the contact line. This elastic Marangoni stress can be positive or negative, depending on the prestretch that is imposed. Without prestretch $\left(\lambda_{\infty}=1\right)$, the contact line region has the largest surface tension, giving a Marangoni stress that is oriented toward the contact line $\left(\sigma_{n t}<0\right)$. Conversely, when the imposed $\lambda_{\infty}$ is large, the contact line region has the smallest surface tension, and the Marangoni stress is directed away from the contact line $\left(\sigma_{n t}>0\right)$. This value is further quantified in Fig. 4, where the change of direction of the Marangoni effect is observed to be close to $\lambda_{\mathrm{cl}} \approx 1.2$. Indeed, this result nearly coincides with the point where $\lambda_{\text {tip }} \approx \lambda_{\infty}$ [cf. Fig. 2(b)]. So the orientation of the Marangoni stress depends on whether the stretch at the tip is larger or smaller than the stretch imposed at a large distance.

\section{EXACT NONLINEAR SOLUTIONS}

\section{A. Splitting off the singularity}

We now pursue a fully analytical theory for the numerical observations above. We see that the elastic singularity is weak, only logarithmic in the stress, so we first try to split off the singularity. For this split, we perform an integration by parts on Eq. (10) by writing

$$
\begin{aligned}
\delta \mathcal{E}= & \int d^{2} X[\operatorname{Div}(\mathbf{s} \cdot \delta \mathbf{x})-(\operatorname{Div} \cdot \mathbf{s}) \cdot \delta \mathbf{x}] \\
& +\oint d S\left(\frac{\partial}{\partial S}(\Upsilon \mathbf{t} \cdot \delta \mathbf{x})-\frac{\partial(\Upsilon \mathbf{t})}{\partial S} \cdot \delta \mathbf{x}\right) .
\end{aligned}
$$

The integral over the third term indeed gives pointlike contributions:

$$
\oint d S \frac{\partial}{\partial S}(\Upsilon \mathbf{t} \cdot \delta \mathbf{x})=-\sum_{\operatorname{disc} i}[\Upsilon \mathbf{t}]_{-}^{+} \cdot \delta \mathbf{x}_{i},
$$

where the sum runs over all possible discontinuities along the contour. The term $\operatorname{Div}(\mathbf{s} \cdot \delta \mathbf{x})$ can be brought to the surface using the divergence theorem. For a smooth domain of integration, the divergence theorem holds for any vector field which is in $\mathcal{L}^{1}$ and whose spatial derivatives are in $\mathcal{L}^{1}$ [47]. This operation is allowed as long as the corresponding singularity is weaker than $1 /|\mathbf{X}|$. Hence, we can write

$$
\begin{aligned}
\delta \mathcal{E}= & -\int d^{2} X \operatorname{Div}(\mathbf{s}) \cdot \delta \mathbf{x}+\oint d S\left(\mathbf{s} \cdot \mathbf{N}-\frac{\partial(\Upsilon \mathbf{t})}{\partial S}\right) \cdot \delta \mathbf{x} \\
& -\sum_{\operatorname{disc} i}[\Upsilon \mathbf{t}]_{-}^{+} \cdot \delta \mathbf{x}_{i} \\
= & -\int d^{2} x \operatorname{div}(\boldsymbol{\sigma}) \cdot \delta \mathbf{x}+\oint d s\left(\boldsymbol{\sigma} \cdot \mathbf{n}-\frac{\partial(\Upsilon \mathbf{t})}{\partial s}\right) \cdot \delta \mathbf{x} \\
& -\sum_{\operatorname{disc} i}[\Upsilon \mathbf{t}]_{-}^{+} \cdot \delta \mathbf{x}_{i},
\end{aligned}
$$

where in the last step we transform the result to the current domain, using the definition of the true stress (or Cauchy stress) according to $\boldsymbol{\sigma}=\mathbf{s} \cdot \mathbf{F}^{T} / \operatorname{det}(\mathbf{F})$.

The condition of equilibrium, $\delta \mathcal{E}=\delta \mathcal{R}$ obtained from Eqs. (9) and (18), then splits into bulk, surface, and point conditions: 


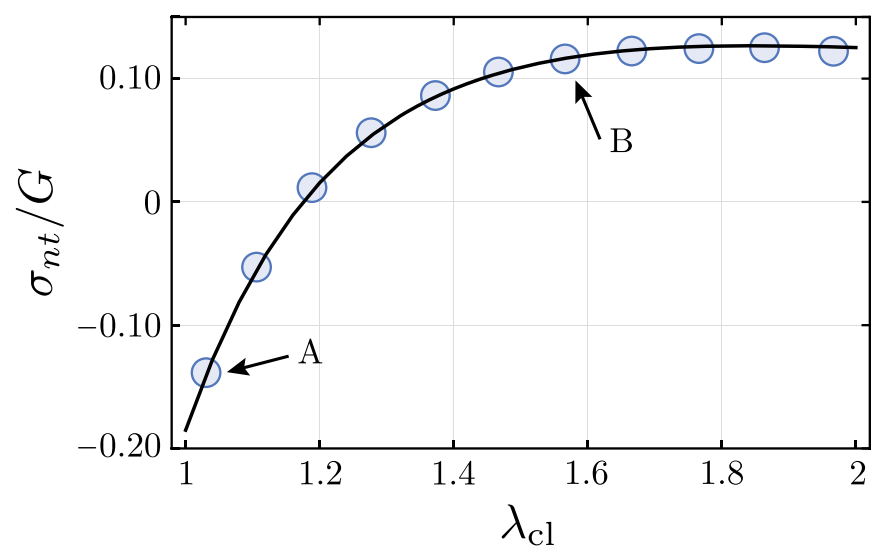

A
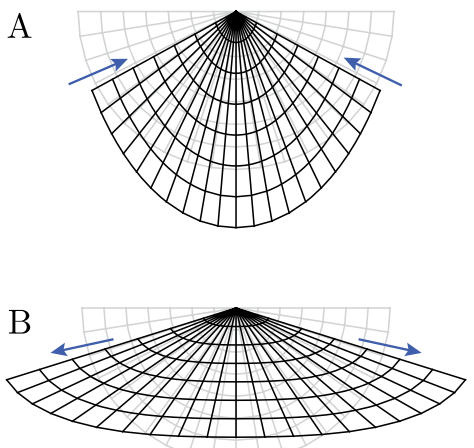

FIG. 4. Marangoni stress $\sigma_{n t} / G$ at the contact line, as a function of the stretch at the contact line $\lambda_{\mathrm{cl}}$. The Marangoni stress can be positive or negative depending on whether the stretch at the tip is larger or smaller than $\lambda_{\infty}$. Open circles are obtained by FEM, the solid line from similarity solutions described in Sec. IV in combination with Neumann's law. The grids represent geometry of the ridge and deformation within it for negative (A) and positive (B) Marangoni stresses, as obtained from the similarity solutions. The gray lines denote the undeformed grid, and the arrows indicate the direction of the Marangoni stress.

$$
\begin{gathered}
\operatorname{div}(\boldsymbol{\sigma})=0, \quad \mathbf{x} \in \mathcal{D}, \\
\boldsymbol{\sigma} \cdot \mathbf{n}-\frac{\partial(\Upsilon \mathbf{t})}{\partial s}=0, \quad \mathbf{x} \in \partial \mathcal{D}, \\
{[\Upsilon \mathbf{t}]_{-}^{+}+\gamma_{\mathrm{LV}} \mathbf{t}_{\mathrm{LV}}=0, \quad \mathbf{x}=\mathbf{x}_{\mathrm{cl}},}
\end{gathered}
$$

where $\mathcal{D}$ denotes the current domain of the deformed state. Besides the classical elastic stress equilibrium in bulk [Eq. (19)], the interface condition [Eq. (20)] gives the Marangoni effect where $\sigma_{n t} \equiv \mathbf{t} \cdot \boldsymbol{\sigma} \cdot \mathbf{n}$ balances gradients in surface tension $\partial \Upsilon / \partial s$, while the normal component of elastic stress $\sigma_{n n} \equiv \mathbf{n} \cdot \boldsymbol{\sigma} \cdot \mathbf{n}$ balances the Laplace pressure. Finally, the Neumann condition appears at the contact line [Eq. (21)], expressed as a discontinuity of the surface tangents. The only assumption made in the derivation above is that the stress singularity is sufficiently weak for the divergence theorem to be applicable, as is the case for a logarithmic singularity.

\section{B. Similarity solutions}

We now analytically establish the nature of the elastic singularity, through an asymptotic analysis near the contact line. For this analysis, we express the mapping $\chi(\mathbf{X})$ in polar coordinates, $(r, \varphi)$ and $(R, \Phi)$, respectively, for the current and reference state. The contact line is located at $r=0$ and $R=0$, and without loss of generality the initially flat free surface is chosen to be along the lines $\Phi=0$ and $\Phi=\pi$. We make use of the fact that the boundary condition (21) forces the solid into an angle $\theta_{S}$, which is defined by the property

$$
\theta_{S}=\lim _{R \rightarrow 0}\left(\varphi_{\Phi=\pi}-\varphi_{\Phi=0}\right)
$$

As is common with singularities [48], we expect the asymptotics to be scale invariant, so we propose a similarity ansatz:

$$
\begin{aligned}
r(R, \Phi) & =R^{\alpha} g_{1}(\Phi), \\
\varphi(R, \Phi) & =R^{\beta} g_{2}(\Phi) .
\end{aligned}
$$

Imposing Eq. (22), one finds that $\beta=0$. A critical feature of soft elastic solids is that these are basically incompressible, i.e., $\operatorname{det}(\mathbf{F})=1$. Combined with $\beta=0$, this result then dictates $\alpha=1$, which implies that the radial stretch $\lambda_{r}=d r / d R$ remains finite and is independent of $R$. In the azimuthal direction, incompressibility implies a relation between the functions $g_{1,2}$, which can be accounted for by writing

$$
\begin{aligned}
r(R, \Phi) & =\frac{R}{\sqrt{f(\Phi)}} \\
\varphi(R, \Phi) & =\int_{\Phi_{0}}^{\Phi} d U f(U),
\end{aligned}
$$

so that the solid angle follows as $\theta_{S}=\int_{0}^{\pi} d \Phi f(\Phi)$. The deformation gradient tensor of this mapping reads

$\mathbf{F}=\left(\begin{array}{ll}F_{r R} & F_{r \Phi} \\ F_{\varphi R} & F_{\varphi \Phi}\end{array}\right)=\left(\begin{array}{cc}\frac{\partial r}{\partial R} & \frac{1}{R} \frac{\partial r}{\partial \Phi} \\ r \frac{\partial \varphi}{\partial R} & \frac{r}{R} \frac{\partial \varphi}{\partial \Phi}\end{array}\right)=\left(\begin{array}{cc}\frac{1}{\sqrt{f}} & -\frac{1}{2 \sqrt{f}} \frac{f^{\prime}}{f} \\ 0 & \sqrt{f}\end{array}\right)$,

which indeed satisfies $\operatorname{det}(\mathbf{F})=1$ for arbitrary $f(\Phi)$. The corresponding Finger tensor reads 


$$
\mathbf{B}=\mathbf{F} \cdot \mathbf{F}^{T}=\left(\begin{array}{cc}
\frac{1}{f}\left[1+\left(\frac{f^{\prime}}{2 f}\right)^{2}\right] & -\frac{f^{\prime}}{2 f} \\
-\frac{f^{\prime}}{2 f} & f
\end{array}\right)
$$

This tensor defines the most general scale-invariant incompressible map that generates a corner.

For the special case where $f^{\prime}=0$, one recovers the classical solution by Singh and Pipkin [49]. However, that solution is shear-free (i.e., $\mathbf{F}$ and $\mathbf{B}$ are diagonal) and, therefore, cannot be universally valid. Here, we derive the most general corner solution that satisfies mechanical equilibrium, $\operatorname{div}(\boldsymbol{\sigma})=0$. We focus on a neo-Hookean material defined by Eq. (11), which has a Cauchy stress $\boldsymbol{\sigma}=G \mathbf{B}-p \mathbf{I}$, so that Eq. (19) becomes

$$
\operatorname{grad}(p)=G \operatorname{div}(\mathbf{B})
$$

This equation implies that $\operatorname{div}(\mathbf{B})$ must be irrotational, i.e., $\operatorname{curl}[\operatorname{div}(\mathbf{B})]=0$, which here takes the form

$$
\frac{\partial B_{r \varphi}}{\partial \varphi}+B_{r r}-B_{\varphi \varphi}=K,
$$

where $K$ is an integration constant. Inserting Eq. (26) and bearing in mind that $\partial / \partial \varphi(\cdots)=(\cdots)^{\prime} / f$, we find

$$
-\left(\frac{f^{\prime}}{2 f}\right)^{\prime}+1+\left(\frac{f^{\prime}}{2 f}\right)^{2}-f^{2}=K f .
$$

This equation is a nonlinear second-order ordinary differential equation for $f(\Phi)$. As boundary conditions, we impose the stretch at each of the boundaries, which subsequently gives the shear stress via the connections

$$
\lambda_{r}=\frac{d r}{d R}=\frac{1}{\sqrt{f(\Phi)}} \Rightarrow \sigma_{r \varphi}=-\frac{G f^{\prime}(\Phi)}{2 f(\Phi)} .
$$

We note that $\lambda_{r}$ in the similarity solution is independent of $R$ and can, therefore, be identified to the stretch at the contact line $\lambda_{r}=\lambda_{\text {cl }}$. The constant $K$ can be adjusted to accommodate the desired $\theta_{S}$. Explicit solutions are presented below and compared directly to FEM simulations.
Once a solution is found, one can explicitly integrate Eq. (27) to obtain the pressure

$$
p(r, \varphi)=G K \log (r / b),
$$

which contains an integration constant $b$ that is fixed by matching to the outer large-scale solution. Thus the analytical description of incompressible corner solutions in the fully nonlinear regime is complete.

\section{Theory compared to FEM}

The similarity solutions derived above capture all FEM results of Sec. III, in the vicinity of the contact line. First, we consider the stress, which for a neo-Hookean solid is given by $\boldsymbol{\sigma}=G \mathbf{B}-p \mathbf{I}$. Our theory explains the FEM result that the normal stress diverges logarithmically, following the singularity of pressure (31), and offers a way to compute the prefactor $K$. Furthermore, the corner solution shows that $\mathbf{B}$ as given in Eq. (26) remains finite at the contact line. This result explains why the Marangoni stress $\sigma_{n t}=\sigma_{r \varphi}$ remains finite at the contact line.

We now turn to a fully quantitative analysis, by solving Eq. (29) for various boundary conditions. Typical (symmetric) similarity solutions are represented graphically in Fig. 5, denoting the Lagrangian grid in both undeformed (gray) and deformed (black) configurations. The three panels each correspond to $\theta_{S}=120^{\circ}$, with different amounts of stretch imposed on the free surfaces. In Fig. 5(a), we report the solution without shear stress, for which $f^{\prime}=0$ for all $\varphi$. In this case, Eq. (24) reduces to the classical solution by Singh and Pipkin [49], with the constant $f=\theta_{S} / \pi$. In the context of elastocapillary ridges, the absence of shear corresponds to a substrate without a Shuttleworth effect. This correspondence explains why, in the absence of a Shuttleworth effect, the stretch at the contact line $\lambda_{\mathrm{cl}}$ is found to be independent of $\lambda_{\infty}$ in our FEM simulations: In a shear-free corner, the stretch takes on a specific value that depends only on the solid angle, as $\lambda_{r}=\lambda_{\mathrm{cl}}=\sqrt{\pi / \theta_{S}}$. The stretch at the contact line is, therefore, locally determined by $\theta_{S}$, irrespective of the conditions imposed at a large distance. Furthermore, in this specific case without shear stress, we find an analytical expression for the strength of the pressure singularity, the
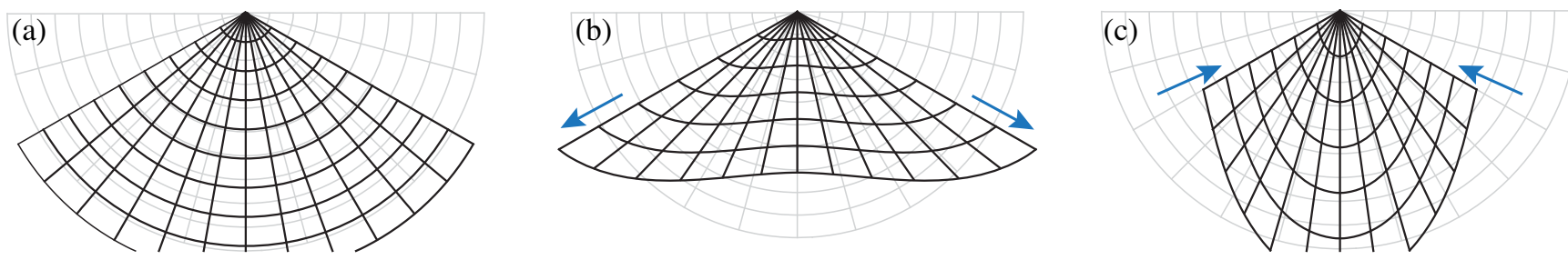

FIG. 5. Similarity solutions for symmetric corners obtained from Eqs. (24) and (29), all with $\theta_{S}=120^{\circ}$. (a) Without the Shuttleworth effect, the shear stress vanishes at the interface, and one recovers the Singh-Pipkin solution [49]. (b),(c) The Shuttleworth effect induces Marangoni stresses, giving positive (b) or negative (c) elastic shear stress at the interface, the direction indicated by the arrows. 
constant $K$ in Eq. (31). Inserting $f=\theta_{S} / \pi$ in Eq. (29) gives $K=\left(\pi / \theta_{S}\right)-\left(\theta_{S} / \pi\right)$. Indeed, this expression is exactly the scaling used in Fig. 3(a), necessary to account for the $\theta_{S}$ dependence, which renders the asymptotes of the curves parallel. Note that the integration constant $b$ in Eq. (31) is not universal, which is why the asymptotes in Fig. 3(a) appear with a horizontal shift. This result demonstrates that the corner solutions are fully quantitative and provide the correct asymptotics observed in FEM, valid in the strongly nonlinear regime.

The Shuttleworth effect dramatically changes the physical picture. Now, a variety of surface stretches $\lambda_{r}$ is possible, as shown in Figs. 5(b) and 5(c). Each of these solutions comes with its own value of the elastic Marangoni stress. Figure 4 illustrates this point, where the prediction of the similarity solutions is shown as a solid line and compared directly to the Marangoni stress in FEM. For the symmetric surface tensions considered in our simulations, the corresponding similarity solution is naturally symmetric and can be found without any adjustable parameters: It follows directly from the surface constitutive relation (12), which in combination with Neumann's law determines the appropriate combination of $\theta_{S}$ and $\lambda$. The perfect prediction of the elastic Marangoni stress in Fig. 4 confirms that the corner solutions indeed offer the correct asymptotic description of the singularity-also in the presence of the Shuttleworth effect.

As a concluding remark, we emphasize again that the observation in PDMS that $\lambda_{\mathrm{cl}}$ increases upon varying $\lambda_{\infty}$ [20] cannot be explained in a hyperelastic theory without a Shuttleworth effect.

\section{LIQUID CONTACT ANGLE, PINNING, AND ESHELBY FORCES}

\section{A. Hysteresis via a process zone}

So far, we consider an isolated contact line, at some prescribed position $\mathbf{X}_{\mathrm{cl}}$, pulling vertically with perfectly symmetric wetting conditions. In a real wetting problem, however, a droplet spreads dynamically until it reaches its equilibrium liquid angle - simultaneously, the contact line reaches an equilibrium material position $\mathbf{X}_{\mathrm{cl}}$, which is not known a priori but which needs to be found selfconsistently. Hence, the full equilibration involves a free exploration of the contact line over the substrate. Technically, such an equilibrium without pinning implies that the change of material coordinate is energetically neutral. Naturally, this equilibration is the case when the substrate is perfectly homogeneous in its reference state. Indeed, in contrast to the rigid case, there are various examples where well-prepared soft polymeric substrates are basically free from pinning and contact angle hysteresis $[20,31,32,50]$.

Here, we take the opposite perspective and consider the possibility that the presence of the contact line itself induces heterogeneity in the material-in its reference state. Even when the originally prepared soft polymeric substrate does not exhibit permanent defects that provide a frozen surface energy landscape, the substrate can develop heterogeneities dynamically, due to the presence of the contact line. Indeed, a large-stress region builds up at a small scale, which can lead to irreversible plastic flow, like in the "fracture process zone" that forms at a crack tip. Although wetting-induced damaging processes are evidenced in experiments where a soft gel exhibits fracture by wetting [51], we focus here on nondamaging plastic deformations in the near-surface region-so that the bulk reference is not affected. Plasticity typically occurs in situations where there is multistability, where multiple stable configurations coexist, which can lead to a hysteretic response upon contact displacement [52]. The large strain may indeed provide a configurational plasticity, without damaging the material. When chains between cross-linkers are long enough to produce entanglement, strain may trigger changes of glassy chain conformation. As an alternative mechanism, the contact line may lead to a local strengthening associated with the elongation of polymeric chains, producing a highly dissipative zone when the contact line explores its environment. Below, we derive the consequences of a nondamaging, plastic process zone induced by the presence of the contact line. By analogy with fracture mechanics, or with defects in crystalline solids, such a plastic process zone can be described by a defect singularity in the theory of elasticity. The singularity then represents the effect of the plastic process zone on the elastic "outer" region. We reveal how the strength of such a defect directly relates to contact angle hysteresis.

\section{B. Displacing an elastocapillary defect}

The consequence of a defect, representing the effect of a process zone on the outer region, can be computed from the change in energy associated to a global displacement of the solution. This consequence is illustrated in Fig. 6, showing such a displacement $\delta \mathbf{X}_{\mathrm{cl}}=\delta U \mathbf{T}$ on the reference domain [Fig. 6(a)] and on the current domain [Fig. 6(b)]. The change in elastic energy associated to the displacement of a defect is known as the Eshelby force [23]:

$$
f_{\mathrm{Esh}}=-\frac{\partial \mathcal{E}_{\mathrm{el}}}{\partial U}=\mathbf{T} \cdot \oint d S \boldsymbol{\Pi} \cdot \mathbf{N},
$$

where the integral encloses the defect and we define the Eshelby's energy-momentum tensor

$$
\boldsymbol{\Pi}=W \mathbf{I}-\mathbf{F}^{T} \cdot \frac{\partial W}{\partial \mathbf{F}} .
$$

The Eshelby force reduces to the $J$ integral in small deformation (linear) elasticity, where it finds an interpretation as the energy release rate in fracture mechanics [53]. 

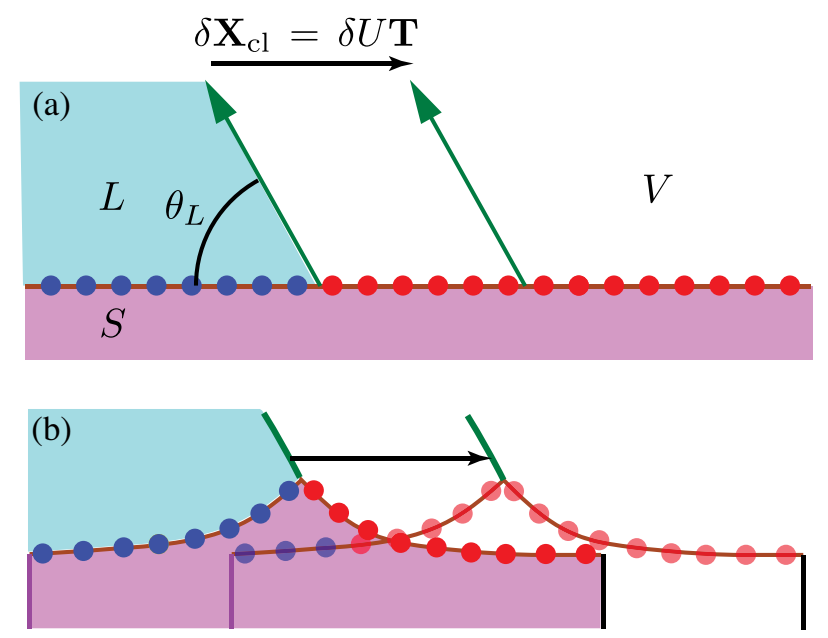

FIG. 6. Determining the liquid contact angle $\theta_{L}$ upon global displacement of the solution. (a) Lagrangian point of view: On the domain of material coordinates, the shift is achieved by a change of material point $\delta \mathbf{X}_{\mathrm{cl}}=\delta U \mathbf{T}$, where the contact line applies. Without pinning, the displacement is energetically neutral, while, in the presence of a pinning defect, an energy $-\Gamma \delta U$ is dissipated at the contact line. (b) Eulerian point of view: The displacement $\delta U$ leads to a variation of the entire solution as given in Eq. (34). At a large distance from the contact line, the change of the surface energies reads $\left(\gamma_{\mathrm{SL}}-\gamma_{\mathrm{SV}}\right) \lambda_{\infty} \delta U$.

To derive the capillary energy released by moving a defect, it is instructive to follow the derivation of the classical result (32), which is based on the application of Noether's theorem in the space of material coordinates [23].

On the reference domain, the displacement simply amounts to a translation $\delta \mathbf{X}_{\mathrm{cl}}=\delta U \mathbf{T}$ of the contact line force, as in Fig. 6(a). The corresponding translation on the current domain is sketched in Fig. 6(b). The idea of deriving the elastic energy released by displacing a defect is to interpret the translation $\delta U$ as a variation $\delta \mathbf{x}$, which can be expressed as

$\delta \mathbf{x}=\chi(\mathbf{X}-\delta U \mathbf{T})-\chi(\mathbf{X})=-\frac{\partial \chi}{\partial \mathbf{X}} \cdot \mathbf{T} \delta U=-\delta U \mathbf{T} \cdot \mathbf{F}^{T}$.

The associated change in elastic energy can be computed from this variation, as

$$
\begin{aligned}
\delta \mathcal{E}_{\mathrm{el}} & =\int d^{2} X \delta \mathbf{x} \cdot\left(\frac{\delta \mathcal{E}_{\mathrm{el}}}{\delta \mathbf{x}}\right)=-\delta U \mathbf{T} \cdot \int d^{2} X\left(\mathbf{F}^{T} \cdot \frac{\delta \mathcal{E}_{\mathrm{el}}}{\delta \mathbf{x}}\right) \\
& =-\delta U \mathbf{T} \cdot \int d^{2} X \operatorname{Div}(\mathbf{\Pi}) .
\end{aligned}
$$

Importantly, in the last step, one uses that the (reference) substrate is homogeneous everywhere except at the defect [23]. When in the vicinity of the singularity $\boldsymbol{\Pi} \sim 1 /\left|\mathbf{X}-\mathbf{X}_{\mathrm{cl}}\right|$, the integral is finite and can be expressed as Eq. (32). When the material is homogeneous everywhere, i.e., no defects, the Eshelby force uniformly vanishes as a consequence of translational invariance.

We now follow the same scheme for the capillary energy, upon replacing $W$ by $\lambda \gamma$, and the deformation gradient tensor $\mathbf{F}$ by its vectorial surface analog $\mathbf{F}_{s}=\partial \mathbf{x}_{s} / \partial S$. Subsequently, we define the surface equivalent of the Eshelby tensor (33), which now is a scalar, and which takes the form

$$
\lambda \gamma-\mathbf{F}_{s}^{T} \cdot\left(\frac{\partial(\lambda \gamma)}{\partial \mathbf{F}_{s}}\right)=\lambda \gamma-\lambda \Upsilon=-\lambda^{2} \gamma^{\prime} \equiv-\mu,
$$

which is the chemical potential anticipated in Eq. (14). Indeed, the associated change in capillary energy reads

$$
\begin{aligned}
\delta \mathcal{E}_{\text {cap }} & =-\delta U \int d S\left(\mathbf{F}_{s}^{T} \cdot \frac{\delta \mathcal{E}_{\text {cap }}}{\delta \mathbf{x}}\right) \\
& =\delta U \int d S \frac{d \mu}{d S}=\delta U[\mu]_{-}^{+},
\end{aligned}
$$

where the integral runs over an infinitesimal domain across the singularity. It is clear that a finite capillary defect energy appears only when $\mu$ exhibits a discontinuity at the contact line, i.e., $[\mu]_{-}^{+} \neq 0$.

We thus conclude that the total energy release rate $\Gamma$, liberated upon displacing the elastocapillary defect at the contact line, takes the form

$$
\Gamma=-\frac{\partial \mathcal{E}}{\partial U}=-[\mu]_{-}^{+}+\mathbf{T} \cdot \oint d S \boldsymbol{\Pi} \cdot \mathbf{N}=-[\mu]_{-}^{+}+f_{\mathrm{Esh}} .
$$

Given that the defect represents a process zone, this result indicates a loss of energy $-\Gamma \delta U$, dissipated inside the process zone during the translation. For the special case where there is no pinning defect and the contact line is free to move, the variation of the contact line position should be energetically neutral, so that $\Gamma=0$.

The notion of the (elastic) Eshelby force in wetting was recently proposed in Ref. [22], where it is argued that the formation of a ridge would already be sufficient to induce an elastic Eshelby force. However, from the above, it is clear that this situation is not the case when the substrate is perfectly homogeneous in its reference state, so that there is a translational invariance of the space of reference coordinates: Applying Noether's theorem to this translational invariance [23], one finds $\partial \mathcal{E}_{\mathrm{el}} / \partial U=0$. This vanishing of the Eshelby force is indeed confirmed by our FEM results and analytical solutions: The stress is only logarithmically singular, so that for an infinitesimal integration volume around the contact line Eq. (32) gives $f_{\text {Esh }}=0$. Therefore, for homogenous substrates, the condition $\Gamma=0$ reduces to $[\mu]_{-}^{+}=0$. The continuity of $\mu$ across the contact line can be interpreted as an "equality of 
chemical potential," necessary for a free exchange of material points across the contact line. This condition of no pinning was previously derived within the strong restrictions of linear elasticity [32] — but it turns out to be valid also when deformations are large.

For a nondamaging process zone, i.e., the reference state remains intact, we expect the Eshelby force to vanish owing to translational invariance. Nonetheless, a capillary defect $\Gamma$ could still emerge, associated with the interfacial microstate of the polymer.

\section{The liquid contact angle}

Up to here, we consider properties of the solid and do not discuss explicitly the liquid. Yet, the liquid contact angle $\theta_{L}$ is the prime feature that characterizes the wetting of a liquid drop. To complete the theory, we now show how the equilibration determines $\theta_{L}$ on homogeneous substratesand how the maximum strength of a contact line defect can be related to contact angle hysteresis on elastic substrates.

We restrict ourselves to the case of a sufficiently large drop, so that far away from the contact line one encounters a flat substrate (Fig. 6). At a large distance from the contact line, the substrate, respectively, has a solid-liquid energy $\gamma_{\mathrm{SL}}\left(\lambda_{\infty}\right)$ and a solid-vapor energy $\gamma_{\mathrm{SV}}\left(\lambda_{\infty}\right)$. The usual argument leading to Young's law for the contact angle amounts to the global horizontal displacement [12]. In the present case, the (Eulerian) displacement reads $\lambda_{\infty} \delta U$, so that the solid capillary energy increases by $\left(\gamma_{\mathrm{SL}}-\gamma_{\mathrm{SV}}\right) \lambda_{\infty} \delta U$, the value of which has to be taken far away from the contact line. This result balances the work $-\gamma_{\mathrm{LV}} \cos \theta_{L} \lambda_{\infty} \delta U$ performed by the liquid-vapor interface, which together gives Young's law. The situation is modified by the presence of a defect: As described above, such a displacement also involves a dissipation inside the process zone, indicating a loss of energy $-\Gamma \delta U$. By consequence, we find a modification of Young's law:

$$
\begin{aligned}
\lambda_{\infty}\left(\gamma_{\mathrm{SL}}-\gamma_{\mathrm{SV}}\right)_{\lambda_{\infty}}+\Gamma & =-\lambda_{\infty} \gamma_{\mathrm{LV}} \cos \theta_{L} \\
\Rightarrow \gamma_{\mathrm{LV}}\left(\cos \theta_{L}-\cos \theta_{Y, \lambda_{\infty}}\right) & =\lambda_{\infty}^{-1}[\mu]_{\mathrm{SL}}^{\mathrm{SV}},
\end{aligned}
$$

where in the second line we anticipate that $f_{\text {Esh }}=0$ (owing to the weak logarithmic elastic singularity). For homogeneous substrates, $\Gamma=0$, and we recover Young's law for the liquid contact angle. We remark that $\theta_{Y}$ is based on the surface energies corresponding to $\lambda_{\infty}$.

The analysis above, in particular, Eq. (39), can be verified by the FEM simulations. In the numerics, we fix a priori the material position $\mathbf{X}_{\mathrm{cl}}$ of the pulling force, so that we effectively work with a pinned contact line. For symmetric surface tensions and pulling vertically, this case is equivalent to the unpinned case, but we can consider any liquid angle $\theta_{L}$, by changing the pulling direction $\mathbf{t}_{\mathrm{LV}}=\left(-\cos \theta_{L}, \sin \theta_{L}\right)$ in Eq. (10). We then measure the jump $[\mu]_{-}^{+}=[\mu]_{\text {SL }}^{\text {SV }}$ across the contact line obtained for the corresponding solution, as a function of $\theta_{L}$. We consider two cases: (i) symmetric surface energies $\gamma_{\mathrm{SL}}=\gamma_{\mathrm{SV}}$ (so that $\theta_{Y}=90^{\circ}$ ) and (ii) asymmetric surface energies $\gamma_{\mathrm{SL}} \neq \gamma_{\mathrm{SV}}$ (here, with $\theta_{Y}=113.6^{\circ}$ ).

The result is presented in Fig. 7(a). It is clear that both cases, symmetric and asymmetric, are in perfect agreement with Eq. (39) with $\Gamma=-[\mu]_{\text {SL }}^{\text {SV }}$. This agreement implies that $f_{\text {Esh }}=0$, consistent with the weak logarithmic singularity. Hence, $\theta_{L}$ can be different from its equilibrium value $\theta_{Y}$ by the presence of a nondamaging process zone, represented by a capillary defect. In that case, interfacial plasticity could be associated with a contact angle hysteresis.
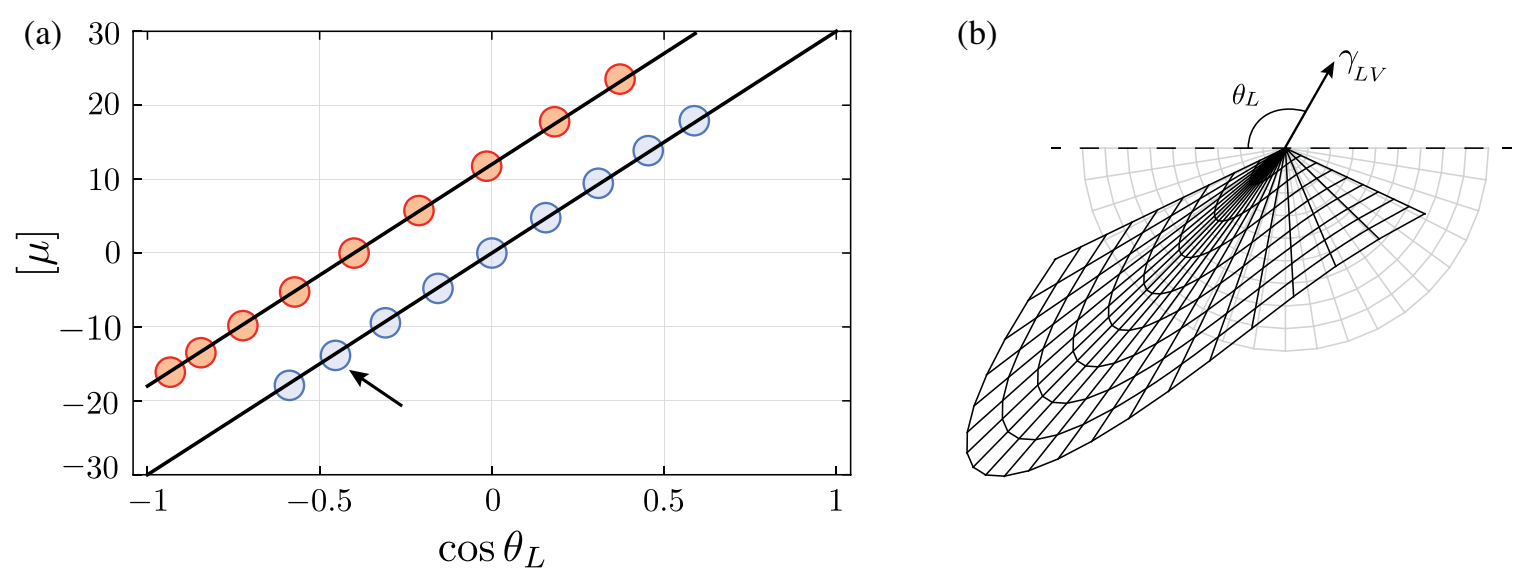

FIG. 7. (a) The strength of the surface defect, quantified by the discontinuity of chemical potential $[\mu]_{-}^{+}$, plotted versus the liquid contact angle $\theta_{L}$. Solid lines are from the theoretical prediction (39), open symbols from FEM with the Shuttleworth effect $\left(c_{0}=c_{1}=1\right)$. Blue data: Symmetric surface energies $\gamma_{0, \mathrm{SV}}=\gamma_{0, \mathrm{SL}}=\gamma_{\mathrm{LV}}$, so that the equilibrium angle $\theta_{Y}=90^{\circ}$. Red data: Asymmetric surface energies $\gamma_{0, \mathrm{SV}}=4 / 5 \gamma_{\mathrm{LV}}$ and $\gamma_{0, \mathrm{SL}}=6 / 5 \gamma_{\mathrm{LV}}$, so that based on $\lambda_{\infty}=1$ we find $\cos \theta_{Y}=-2 / 5$. The numerics confirm that $[\mu]_{-}^{+}$provides the pinning force; when pulling at $\theta_{L}=\theta_{Y}$, there is no pinning and $[\mu]_{-}^{+}=0$. (b) The grid plot represents the asymmetric ridge for symmetric surface energies, resulting from a contact angle $\theta_{L}>\theta_{Y}$. This ridge corresponds to the data point marked by an arrow in the main panel. 
A typical asymmetric similarity solution is shown via the grid representation in Fig. 7(b), for which there is a jump in stretch across the contact line. We remark that, in all cases, Neumann's law is still observed to be valid, irrespective of the defect.

\section{DISCUSSION}

In summary, we explore analytically and numerically the macroscopic theory for elastocapillary ridges, based on the minimization of a bulk elastic free energy and a surface capillary free energy. This theory offers a fully nonlinear description of soft wetting, including the possibility that capillarity depends on strain (Shuttleworth effect), large elastic deformation, and pinning. In this macroscopic theory, there is a perfect separation of scales between elastocapillary length $\gamma / G$ and the molecular scale $a$, since effectively $a \rightarrow 0$ in the continuum. This limit is relevant for typical experiments, and it is of theoretical importance in order to reveal the nature of the ridge singularity as predicted from large deformation elasticity. We now discuss these new theoretical results in comparison to recent literature on the Shuttleworth effect.

\section{A. Theory \\ 1. First boundary condition}

In this macroscopic description, it is found that the stress singularity associated with the contact line ridge is weak (i.e., logarithmic) and, therefore, integrable, under all conditions that are considered. Hence, the singularity does not behave analogously to an elastic disclination defect, and no qualitative difference emerges when the substrate is globally stretched. As a consequence, in this limit where $\gamma / G a \rightarrow \infty$, the Neumann tension balance at the contact line is strictly valid. In the scheme of energy minimization, Neumann's law emerges as auxiliary condition (21) and as such serves as a first boundary condition at the contact line. We have no explanation for why previous continuum simulations suggest a deviation from Neumann's law $[21,22]$. We emphasize, however, that the present numerics are based on an adaptive method, which is necessary to fully resolve the elastic singularity, and that we extensively verify that the results are fully converged. Furthermore, we derive new analytical solutions of nonlinear elasticity that describe the singularity - these are indeed perfectly recovered by the numerics.

How can one understand the deviation from Neumann's law observed in molecular dynamics simulations of wetting on cross-linked polymer networks [33]? This deviation finds its origin in the lack of scale separation between $\gamma / G$ and the molecular scale $a$, which is inevitable in molecular simulations-the scale $a$ there enters as a molecular cutoff of the continuum and also gives a finite width of the interface. As argued in Refs. [26,27], the elastic contributions near the contact line can be computed by integrating the elastic stress over a small but finite region-in molecular simulations, the smallest possible size for this region would be $a$. In the present work, we demonstrate that the stress singularity is always logarithmic: $\sigma \sim G \log [r /(\gamma / G)]$. Hence, the integral over stress gives an elastic contribution

$$
\int_{-a}^{a} d r \sigma \sim G a \log (a G / \gamma)
$$

which needs to be compared to the surface tensions. In molecular simulations, where typically $\gamma / G a$ is of the order of 1-100, a measurable elastic correction to Neumann's law indeed appears. We refer to Ref. [27] for a quantitative test of Eq. (40), as the elastic correction to Neumann's law. However, in typical experiments performed with polymeric gel, where the $\gamma / G$ is well above the micron scale, the scale-separation correction is $10^{-4}$, and one approaches the macroscopic continuum limit. In such experiments, one safely concludes that Neumann's law holds.

\section{Second boundary condition versus pinning}

While much theoretical work focuses on the validity (or not) of Neumann's law, very little attention is given to the implications of contact line pinning [27]. In many experiments on soft polymer networks, contact line pinning is virtually absent, as quantified by a very small hysteresis $[20,31,32,50]$. This absence implies that the contact line can freely move, exchanging the substrate's material point touching the liquid-vapor interface without any energetic cost. Here, we demonstrate that such a free motion occurs only under a very specific condition. Namely, the chemical potential defined by $\mu=\lambda^{2} d \gamma / d \lambda$ must be continuous across the contact line. This continuity of chemical potentials is the second boundary condition that needs to be imposed when there is no contact line pinning. Such a condition was previously derived under the restrictive assumption of linear elasticity [32] — here, we demonstrate this assumption to be valid also at large deformation and explore its consequences in numerical simulations. In particular, we confirm numerically that Young's law is recovered only when the second boundary condition $\mu_{\mathrm{SV}}=$ $\mu_{\mathrm{SL}}$ is satisfied at the contact line. For asymmetric surface energies, the second boundary condition, in general, implies a jump in stretch across the contact line, so that in the presence of a Shuttleworth effect one generically expects large deformations.

The possibility of pinning is interesting in itself. Depending on the material strength, large deformations might lead to fracture, as observed in Ref. [51], or local plasticity. We demonstrate how such a local "process zone" can be accounted for by introducing a defect in the elastocapillary continuum theory. With the defect, one can accommodate a range of angles $\theta_{L}$ by adjusting the strength of the defect at the contact line. In our simulations, 
we encounter only a weak logarithmic singularity of elastic stress, which implies that the strength of the defect received no contribution from elasticity (i.e., the elastic Eshelby force vanishes). The defect strength, in fact, is found to be equal to the discontinuity in chemical potential at the contact line, i.e., $\Gamma=-[\mu]_{-}^{+}$, giving rise to a modified Young's law (39). In practice, one expects the defect to exhibit a "toughness," just like in fracture, which is the maximum value that can be sustained before depinning occurs. Given Eq. (39), we immediately infer that this argument implies a contact angle hysteresis, with advancing and receding angles $\cos \theta_{r}-\cos \theta_{a}=2\left|[\mu]_{-}^{+}\right|_{\max }$. Future theoretical work should be dedicated to a more detailed description of the interior of the process zone.

\section{Beyond the incompressible neo-Hookean solid}

The numerical simulations and the analysis above are performed for an incompressible solid with a neo-Hookean constitutive relation. However, reticulated polymer networks may exhibit an elasticity different from this idealization $[32,54,55]$. While the neo-Hookean solid brings out all the kinematic nonlinearities associated with large deformations, it is important to confirm to what extent the results are robust with respect to different choices of the solid's constitutive relation.

A beautiful feature of the shear-free mapping by Singh and Pipkin [49], Eq. (24) with a constant $f$, is that it is an exact solution for an arbitrary incompressible hyperelastic solid, regardless of its constitutive law. In fact, this feature is what made the solution of particular interest to Ref. [49], since it can be used for the purpose of material characterization. We thus conclude that for the incompressible corner without shear, i.e., without the Shuttleworth effect, the corner solution applies to any hyperelastic solid. Hence, the kinematics, in particular, the stretch at the contact line $\lambda_{\mathrm{cl}}=\sqrt{\pi / \theta_{S}}$, is universal. The asymptotics for the pressure (31) are also unaffected-only the relation between the prefactor $K$ and the solid angle $\theta_{S}$ changes for different constitutive relations.

For the case with a Shuttleworth effect, i.e., Eq. (24) with $f^{\prime} \neq 0$ so that there is shear, the solution is no longer independent of the solid's constitutive relation. Importantly, however, the kinematic structure that we identify, as expressed by Eq. (26), is still valid for any hyperelastic solid. This validity means that the stretches remain finite and are independent of the radial distance $r$ to the contact line. One can show that the corresponding pressure is again logarithmic, still following the asymptotics (31).

Hence, for incompressible solids, changing the solid's constitutive relation does not alter the kinematics nor the nature of the stress singularity. Though elastomers are typically incompressible, we numerically verify that the stress singularity is still logarithmic for a compressible solid at finite deformation (using a compressible neoHookean model, with Poisson ratio 0.4). The central conclusions of our paper, regarding the logarithmic stress singularity at large deformations and its consequences for the interpretation of experiments, are, thus, generally valid, beyond the incompressible neo-Hookean solid.

\section{B. Experiments and outlook}

Experiments that probe the strain dependence of surface tension are so far based on wetting experiments, with a key role to the contact angles of the solid and of the liquid. Having established the elastocapillary continuum framework for soft wetting, for the first time consistently accounting for large deformations and the Shuttleworth effect, we can now critically assess the experimental situation.

Different series of experiments have been performed with stretched PDMS gels, for static $[20,30]$ and dynamical wetting [32]. They consistently show a change of the solid angle $\theta_{S}$ under stretching. Similarly, the solid angle is found to change in dynamical experiments on Polyvinylsiloxane [35]. Our results consolidate these experimental results: In the Appendix, we show at what scale the "true" Neumann angle $\theta_{S}$ is reached and confirm that these lie within the experimental resolution. To date, these experimental observations have not received any other explanation than via a surface tension that depends on the strain (or on the history of strain). Hence, they offer a convincing case for a nontrivial surface constitutive relation in soft polymer networks, at least for two different systems.

Another direct piece of evidence for the Shuttleworth effect is that experiments in Ref. [20] reveal an increase of stretch at the contact line upon a global stretching of the substrate. This information was previously not used to interpret results in the context of a Shuttleworth effect. However, our numerical and analytical results show that such a variation of the stretch at the contact line can occur only in the presence of elastic Marangoni stresses, induced by a Shuttleworth effect-if surface tension were constant, the stretch at the contact line would take on a constant value.

This result evokes an important question that remains to be resolved: What is the microscopic origin of the coupling between surface energy and strain? The polymer is expected to be liquidlike at a small scale, where surface tension is exerted: What can produce the coupling between the microscopic scale and the deformation of the network of reticulation (or entanglement) points? A possible scenario is that the coupling emerges from a superficial layer where the mechanical and structural properties are different from the bulk [25,31]. Related to this open question is the experimental observation that, in contrast to solid angle $\theta_{S}$, the liquid contact angle $\theta_{L}$ turns out not to depend on stretching [31] - a property that is confirmed for six different liquid-substrate systems in Ref. [31] and which also holds for PDMS [20,32], for substrates stretched up to $100 \%$. This result is surprising, since Young's law for the liquid angle should be valid for sufficiently large drops, but with surface energies $\gamma_{\mathrm{SV}}-\gamma_{\mathrm{SL}}$ based on the externally 
imposed stretch $\lambda_{\infty}$ [31]. This interpretation of Young's law is confirmed in Sec. V, in an analysis where the large deformation elasticity and the Shuttleworth effect are explicitly accounted for. The implication of the experimental invariance of $\theta_{L}$ (within an experimental resolution of $\pm 1^{\circ}$ ) is that, for all imposed $\lambda_{\infty}$, the strain dependence $d \gamma / d \lambda$ must be nearly the same on both sides of the contact line. While there is no understanding of the microscopic or mesoscopic origin of the strain-dependent surface energy, there is a fortiori no real understanding of this property, observed to be valid for many different pairs of liquid and reticulated polymers. However, it indicates that the contribution to the surface energy which depends on strain, and, therefore, on the deformation of the reticulation points network, is the same with and without a liquid in contact. The situation is very different for glassy polymers, for which it is found that the polarity of the liquid affects $d \gamma / d \lambda$ [31]. We infer that, for elastomers, the strain dependence of excess energy in the superficial layer must be associated with an anomalous elastic property and not with the interaction between the polymeric surface and the fluid.

Another assessment of the Shuttleworth effect makes use of an elastic Wilhelmy plate, where a polymeric wire is partially immersed in a liquid reservoir-allowing one to measure the stretch discontinuity across the contact line. In Ref. [34], it is found that the strain remains very small and no discontinuity is observed-implying once more that $d \gamma / d \lambda$ is equal on both sides. In the initial experiment in Ref. [28], conversely, a strong discontinuity of strain is observed at the contact line, implying a jump in $d \gamma / d \lambda$. Given that strains remain very small in these experiments, we can assume that the measured strain reflects the actual strains close to the contact line. Therefore, one can interpret these experiments using the no-pinning condition of Sec. V, i.e., $[\mu]_{-}^{+}=0$, which at small strains implies the continuity of $d \gamma / d \lambda$ across the contact line-in perfect agreement with the observation in Ref. [34]. It is argued in Ref. [34] that discontinuous strains could be an artifact due to swelling. As an alternative interpretation, we note that in Ref. [28] a strong contact angle hysteresis is observed, which in the Shuttleworth interpretation would also be consistent with a breakdown of the no-pinning condition $[\mu]_{-}^{+}=0$.

This research opens the promising perspective of identifying different conditions or different preparation protocols to get, or not, polymer networks with intricate surface properties. The main open question is to understand the microscopic origin of the Shuttleworth effect, which in the present understanding is confirmed for at least two different systems. We emphasize that, mechanically, none of the experimental observations are in contradiction with the presence of a Shuttleworth effect, in particular, since $\theta_{L}$ and the elastic Wilhelmy plate probe only the difference of strain dependence on either side of the contact line. By contrast, the independent measurements of both the solid angle and the stretch at the contact line [20,30] cannot be explained by a hyperelastic theory without explicitly accounting for a strong Shuttleworth effect. Future experiments on a broad class of soft materials should, therefore, simultaneously explore both contact angles $\theta_{L}$ and $\theta_{S}$, as well as the strains near the contact line. Special attention should be paid on the influence of both the surface functionalization and the surface architecture of the reticulated polymer network on both surface tension and its variation with respect to $\lambda$. The development of molecular dynamics simulations of reticulated polymers may help understand in detail the relation between the Shuttleworth effect and statistical effects in the surface. Combined with the fully nonlinear numerics as presented here, this understanding will offer a systematic quantification of the capillarity of soft solids. A next step is to extend the numerical method to wetting dynamics, for which the solid angle (and, thus, the Shuttleworth effect) is a key parameter. Indeed, a stretched substrate can exhibit an enhanced wetting mobility [32]. A possible route is to simulate contact lines that move at constant velocity. Such a motion generically implies large deformations that excite the substrate's bulk viscoelasticity and possibly historydependent surface rheology [56].

\section{CONCLUSION}

In this article, we have addressed some of the hotly debated questions on soft wetting and its implications for surface mechanics of polymeric networks. The central issues pertained to the nature of the singularity of the elastocapillary ridge, which appears in response to the nanoscopic forcing at a three-phase contact line. Specifically, there has been a controversy on the roles of bulk (elasticity) and surface (capillarity) forces in this singular force balance. Using a combination of asymptotic methods and goal-adaptive finite-element simulations, we revealed that the bulk elastic stress singularity is integrable, irrespective of the solid's constitutive relation. Thereby, bulk elasticity cannot affect the force balance at the contact line-by consequence, the mechanics at the ridge tip involves only capillary forces. In addition, we explored the consequences of plastic events near the highly strained ridge and showed how these relate to the classical Young's law on soft substrates.

The resolution of the controversy allows us to interpret recent measurements in a unified manner. The experimental observations, that the solid ridge angle and its stretch at the contact line vary with an externally applied global stretch, consistently imply a strong Shuttleworth effect: The solid interface represents a surface elasticity where capillary forces depend on the local amount of stretch. Most concretely, our results imply that solid surface tensions can be determined by measuring ridge angles and the local surface stretches. The experimental observation that the liquid angles do not change with a global stretch implies that this surface elasticity is insensitive to the presence of a 
liquid phase in contact with the reticulated polymer. A complete technical review of the state of the art, in relation to our findings, can be found in the discussion in Sec. VI.

More generally, this paper provides a rigorous theoretical framework to characterize the surface elasticity of soft solids-allowing for large deformations and even singularities. It contributes to the promising domain of polymer surface science by a macroscopic analysis of the coupling between adhesion and elasticity. Technological applications in soft microtechnology, ranging from electronics to focal adhesion of biological cells, will have to finely control the chemical functionalization, the polymeric network architecture, and the electronic properties at the surface of reticulated polymer.

\section{ACKNOWLEDGMENTS}

We thank J. Eggers, M. van Gorcum, T. Salez, and R. Style for discussions. We acknowledge financial support from European Research Council Consolidator Grant No. 616918 (to A.P. and S. K.), from French National Agency for Research grant SMART (to B. A.), from NWO through VICI Grant No. 680-47-632 (to J. H. S.), and an Industrial Partnership Program (a joint research program of Canon Production Printing, Eindhoven University of Technology, University of Twente, and NWO) (to E. H. v. B.).

\section{APPENDIX: ASYMPTOTIC APPROACH TO NEUMANN ANGLE AND EXPERIMENTAL ACCURACY}

The corner solutions described in Sec. IV are valid asymptotically close to the tip. A question of experimental relevance is how rapidly this asymptotic result is approached, specifically, what spatial resolution is required in order to accurately resolve the true, asymptotic ridge angle $\theta_{S}$. Here, we consolidate the experimental method by considering the approach to symmetric corners, from the FEM numerics and from a perturbative analysis based on the corner solutions.

In Fig. 8, we plot the local angle of the interface $\varphi(r)$ (measured with respect to the horizontal, defined as a positive quantity), as it approaches its value at the contact line $\varphi_{\mathrm{cl}}$. For symmetric corners, the solid angle $\theta_{S}=\pi-2 \varphi_{\mathrm{cl}}$. This ultimate angle is approached as

$$
\varphi(r) \simeq \varphi_{\mathrm{cl}}+K \tilde{r} \ln (B \tilde{r}), \quad \text { with } \quad \tilde{r}=\frac{r}{\gamma / G},
$$

as indicated by the red dashed line in Fig. 8. In this expression, $K$ is the same as in Eq. (31), while $B$ depends on the outer solution. The asymptotics (A1) can be obtained from a perturbation analysis around the straight corner. For this analysis, we balance the leading-order elastic stress $p \sim K G \ln (r / b)$ with the Laplace pressure $p \sim \gamma \partial \varphi / \partial r$. This balance indeed gives Eq. (A1) and perfectly describes the asymptotics of the FEM data.

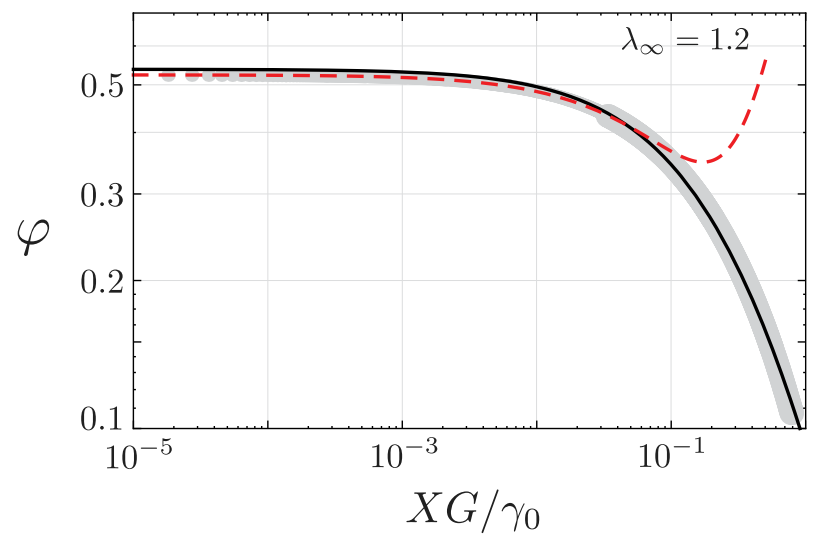

FIG. 8. Typical example of the approach of the Neumann angle: the interface angle $\varphi$ with respect to the horizontal as a function of the scaled distance to the contact line $r G / \gamma$. Light gray symbols correspond to FEM without a Shuttleworth effect $\left(c_{0}=c_{1}=0\right.$, with $\gamma_{0}=\gamma_{\mathrm{LV}}$ ); large symbols represent a typical range accessible to experiments. The red dashed line gives the asymptotics (A1), while the solid black line (A2) gives an improved fit at the scale $r \sim \gamma / G$. The latter is fitted over the range typically accessible in experiment (large symbols, appearing as a thick gray line).

In the experiments of $\mathrm{Xu}$ et al. [20], the spatial resolution of the interface profile is typically $1 \mu \mathrm{m}$, with an elastocapillary length $\gamma / G$ of about $30 \mu \mathrm{m}$. According to the numerical result in Fig. 8, taking the value of $\varphi$ at $r G / \gamma=$ $1 / 30$ typically leads to an overestimation of $\theta_{S}$ by about $10^{\circ}$ (the typical error is the same for all tested $\lambda_{\infty}$ ). Such a value is comparable to the error bar already given in Ref. [20], so that our results consolidate the experimental characterization of the ridge angle. Specifically, it consolidates that $\theta_{S}$ exhibits a strong dependence on the imposed prestretch. We suggest that the experimental resolution can be further improved by fitting the profiles by the asymptotics. Rather than Eq. (A1), which exhibits little overlap in the experimentally accessible range, we propose the form

$$
\varphi(r) \simeq \varphi_{\mathrm{cl}}+K \tilde{r} \ln \left(\frac{B \tilde{r}}{1+\tilde{r}}\right), \quad \text { with } \quad \tilde{r}=\frac{r}{\gamma / G}
$$

This form is shown as the solid line in Fig. 8, fitted over a typical experimental range $\tilde{r}=0.03 \ldots 1$ (large symbols). This form has the correct asymptotics but also provides a good fit at the scale $r \sim \gamma / G$.

[1] J. Li, A. D. Celiz, J. Yang, Q. Yang, I. Wamala, W. Whyte, B. R. Seo, N. V. Vasilyev, J. J. Vlassak, Z. Suo, and D. J. Mooney, Tough Adhesives for Diverse Wet Surfaces, Science 357, 378 (2017).

[2] N. J. Shirtcliffe, G. McHale, and M. I. Newton, The Superhydrophobicity of Polymer Surfaces: Recent Developments, J. Polym. Sci., Part B: Polym. Phys. 49, 1203 (2011). 
[3] J. A. Rogers, T. Someya, and Y. Huang, Materials and Mechanics for Stretchable Electronics, Science 327, 1603 (2010).

[4] D. Discher, P. Janmey, and Y. Wang, Tissue Cells Feel and Respond to the Stiffness of Their Substrate, Science 310, 1139 (2005).

[5] S. Douezan, J. Dumond, and F. Brochard-Wyart, Wetting Transitions of Cellular Aggregates Induced by Substrate Rigidity, Soft Matter 8, 4578 (2012).

[6] D. R. King, M. D. Bartlett, C. A. Gilman, D. J. Irschick, and A. J. Crosby, Creating Gecko-like Adhesives for Real World Surfaces, Adv. Mater. 26, 4345 (2014).

[7] Z. Zou, C. Zhu, Y. Li, X. Lei, W. Zhang, and J. Xiao, Rehealable, Fully Recyclable, and Malleable Electronic Skin Enabled by Dynamic Covalent Thermoset Nanocomposite, Sci. Adv. 4, eaaq0508 (2018).

[8] K. Binder and W. Kob, Glassy Materials and Disordered Solids: An Introduction to Their Statistical Mechanics (World Scientific, Singapore, 2011).

[9] P.-G. De Gennes, Scaling Concepts in Polymer Physics (Cornell University, Ithaca, 1979).

[10] M. Doi and S. F. Edwards, The Theory of Polymer Dynamics (Oxford University, New York, 1988), Vol. 73.

[11] M. Rubinstein, R. H. Colby et al., Polymer Physics (Oxford University, New York, 2003), Vol. 23.

[12] P.-G. de Gennes, F. Brochard-Wyart, and D. Quéré, Capillarity and Wetting Phenomena: Drops, Bubbles, Pearls, Waves (Springer, Berlin, 2002).

[13] A. Rusanov, Theory of Wetting of Elastically Deformed Bodies. 1. Deformation with a Finite Contact-Angle, Colloid J. USSR 37, 614 (1975).

[14] M. Shanahan, The Influence of Solid Micro-deformation on Contact-Angle Equilibrium, J. Phys. D 20, 945 (1987).

[15] A. Carré, J.-C. Gastel, and M. E. R. Shanahan, Viscoelastic Effects in the Spreading of Liquids, Nature (London) 379, 432 (1996).

[16] L. White, The contact Angle on an Elastic Substrate. 1. The Role of Disjoining Pressure in the Surface Mechanics, J. Colloid Interface Sci. 258, 82 (2003).

[17] R. Pericet-Camara, A. Best, H. J. Butt, and E. Bonaccurso, Effect of Capillary Pressure and Surface Tension on the Deformation of Elastic Surfaces by Sessile Liquid Microdrops: An Experimental Investigation, Langmuir 24, 10565 (2008).

[18] E. R. Jerison, Y. Xu, L. A. Wilen, and E. R. Dufresne, Deformation of an Elastic Substrate by a Three-Phase Contact Line, Phys. Rev. Lett. 106, 186103 (2011).

[19] S. Park, B. Weon, J. Lee, J. Lee, J. Kim, and J. Je, Visualization of Asymmetric Wetting Ridges on Soft Solids with X-Ray Microscopy, Nat. Commun. 5, 4369 (2014).

[20] Q. Xu, K. Jensen, R. Boltyanskiy, R. Sarfat, R. W. Style, and E. R. Dufresne, Direct Measurement of Strain-Dependent Solid Surface Stress, Nat. Commun. 8, 555 (2017).

[21] H. Wu, Z. Liu, A. Jagota, and C.-Y. Hui, Effect of Large Deformation and Surface Stiffening on the Transmission of a Line Load on a Neo-Hookean Half Space, Soft Matter 14, 1847 (2018).

[22] R. Masurel, M. Roché, L. Limat, I. Ionescu, and J. Dervaux, Elastocapillary Ridge as a Noninteger Disclination, Phys. Rev. Lett. 122, 248004 (2019).
[23] J. D. Eshelby, The Elastic Energy-Momentum Tensor, J. Elast. 5, 321 (1975).

[24] R. Shuttleworth, The Surface Tension of Solids, Proc. Phys. Soc. London Sect. A 63, 444 (1950).

[25] B. Andreotti and J. H. Snoeijer, Soft Wetting and the Shuttleworth Effect, at the Crossroads between Thermodynamics and Mechanics, Europhys. Lett. 113, 66001 (2016).

[26] R. W. Style, A. Jagota, C.-Y. Hui, and E. R. Dufresne, Elastocapillarity: Surface Tension and the Mechanics of Soft Solids, Annu. Rev. Condens. Matter Phys. 8, 99 (2017).

[27] B. Andreotti and J. H. Snoeijer, Statics and Dynamics of Soft Wetting, Annu. Rev. Fluid Mech. 52, 285 (2020).

[28] A. Marchand, S. Das, J. H. Snoeijer, and B. Andreotti, Capillary Pressure and Contact Line Force on a Soft Solid, Phys. Rev. Lett. 108, 094301 (2012).

[29] J. Bostwick, M. Shearer, and K. Daniels, Elastocapillary Deformations on Partially-Wetting Substrates: Rival Contact-Line Models, Soft Matter 10, 7361 (2014).

[30] Q. Xu, R. W. Style, and E. R. Dufresne, Surface Elastic Constants of a Soft Solid, Soft Matter 14, 916 (2018).

[31] R. D. Schulman, M. Trejo, T. Salez, E. Raphaël, and K. Dalnoki-Veress, Surface Energy of Strained Amorphous Solids, Nat. Commun. 9, 982 (2018).

[32] J. H. Snoeijer, E. Rolley, and B. Andreotti, Paradox of Contact Angle Selection on Stretched Soft Solids, Phys. Rev. Lett. 121, 068003 (2018).

[33] H. Liang, Z. Cao, Z. Wang, and A. V. Dobrynin, Surface Stresses and a Force Balance at a Contact Line, Langmuir 34, 7497 (2018).

[34] S.-Y. Chen, A. Bardall, M. Shearer, and K. E. Daniels, Distinguishing Deformation Mechanisms in Elastocapillary Experiments, Soft Matter 15, 9426 (2019).

[35] M. van Gorcum, S. Karpitschka, B. Andreotti, and J. H. Snoeijer, Spreading on Viscoelastic Solids: Are Contact Angles Selected by Neumann's Law?, Soft Matter 16, 1306 (2020).

[36] E. van Brummelen, M. S. Roudbari, G. Simsek, and K. van der Zee, Binary-Fluid-Solid Interaction Based on the Navier-Stokes-Cahn-Hilliard Equations, in Fluid Structure Interaction, edited by S. Frei, B. Holm, T. Richter, T. Wick, and H. Yang (De Gruyter, Berlin, 2017), pp. 283-328.

[37] L. A. Lubbers, J. H. Weijs, L. Botto, S. Das, B. Andreotti, and J. H. Snoeijer, Drops on Soft Solids: Free Energy and Double Transition of Contact Angles, J. Fluid Mech. 747, R1 (2014).

[38] Note that in the present work the solid interface is treated as part of the substrate, so that the external traction of the liquid-vapor interface is $\gamma_{\mathrm{LV}} \mathbf{t}_{\mathrm{LV}}$. The force transmitted onto the elastic bulk of the substrate, i.e., after passing the interface, is more intricate as discussed, e.g., in Refs. [25,28,29].

[39] R. W. Style and E. R. Dufresne, Static Wetting on Deformable Substrates, from Liquids to Soft Solids, Soft Matter $\mathbf{8}$, 7177 (2012).

[40] L. Limat, Straight Contact Lines on a Soft, Incompressible Solid, Eur. Phys. J. E 35, 134 (2012).

[41] R. W. Style, R. Boltyanskiy, Y. Che, J. S. Wettlaufer, L. A. Wilen, and E. R. Dufresne, Universal Deformation of Soft Substrates near a Contact Line and the Direct Measurement 
of Solid Surface Stresses, Phys. Rev. Lett. 110, 066103 (2013).

[42] R. Becker and R. Rannacher, An Optimal Control Approach to a Posteriori Error Estimation in Finite Element Methods, Acta Numer. 10, 1 (2001).

[43] J. Oden and S. Prudhomme, Goal-Oriented Error Estimation and Adaptivity for the Finite Element Method, Comput. Math. Appl. 41, 735 (2001).

[44] R. Nochetto and A. Veeser, Multiscale and Adaptivity: Modeling, Numerics and Applications (Springer, Berlin, 2012), Chap. Primer of Adaptive Finite Element Methods, pp. $125-225$.

[45] E. van Brummelen, S. Zhuk, and G. van Zwieten, WorstCase Multi-objective Error Estimation and Adaptivity, Comput. Methods Appl. Mech. Eng. 313, 723 (2017).

[46] G. van Zwieten, J. van Zwieten, C. Verhoosel, E. Fonn, T. van Opstal, and W. Hoitinga, Nutils (version 5.0).

[47] M. Willem, Functional Analysis, Cornerstones (Springer, New York, 2013).

[48] J. Eggers and M. A. Fontelos, Singularities: Formation, Structure, and Propagation, Cambridge Texts in Applied Mathematics (Cambridge University Press, Cambridge, England, 2015).
[49] M. Singh and A. C. Pipkin, Note on Ericksen's Problem, Z. Angew Math. Phys. 16, 706 (1965).

[50] R. Lhermerout, H. Perrin, E. Rolley, B. Andreotti, and K. Davitt, A Moving Contact Line as a Rheometer for Nanometric Interfacial Layers, Nat. Commun. 7, 12545 (2016).

[51] J. B. Bostwick and K. E. Daniels, Capillary Fracture of Soft Gels, Phys. Rev. E 88, 042410 (2013).

[52] C. Caroli and P. Nozières, Hysteresis and Elastic Interactions of Microasperities in Dry Friction, Eur. Phys. J. B 4, 233 (1998).

[53] J. R. Rice, A Path Independent Integral and the Approximate Analysis of Strain Concentration by Notches and Cracks, J. Appl. Mech. 35, 379 (1968).

[54] K. Urayama, T. Kawamura, and S. Kohjiya, StructureMechanical Property Correlations of Model Siloxane Elastomers with Controlled Network Topology, Polymer 50, 347 (2009).

[55] C. Creton, 50th Anniversary Perspective: Networks and Gels: Soft but Dynamic and Tough, Macromolecules 50, 8297 (2017).

[56] M. van Gorcum, B. Andreotti, J. H. Snoeijer, and S. Karpitschka, Dynamic Solid Surface Tension Causes Droplet Pinning and Depinning, Phys. Rev. Lett. 121, 208003 (2018). 